\title{
Energy and the AdS/CFT Correspondence
}

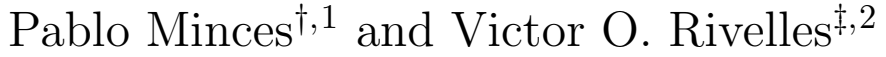 \\ ${ }^{\dagger}$ Instituto de Física Teórica, Universidade Estadual Paulista \\ Rua Pamplona 145, 01405-900, São Paulo, SP, Brasil \\ ${ }^{\ddagger}$ Instituto de Física, Universidade de São Paulo \\ Caixa Postal 66.318, 05315-970, São Paulo, SP, Brasil
}

\begin{abstract}
We consider a scalar field theory on AdS in both minimally and non-minimally coupled cases. We show that there exist constraints which arise in the quantization of the scalar field theory on AdS which cannot be reproduced through the usual AdS/CFT prescription. We argue that the usual energy, defined through the stress-energy tensor, is not the natural one to be considered in the context of the AdS/CFT correspondence. We analyze a new definition of the energy which makes use of the Noether current corresponding to time displacements in global coordinates. We compute the new energy for Dirichlet, Neumann and mixed boundary conditions on the scalar field and for both the minimally and non-minimally coupled cases. Then, we perform the quantization of the scalar field theory on AdS showing that, for 'regular' and 'irregular' modes, the new energy is conserved, positive and finite. We show that the quantization gives rise, in a natural way, to a generalized AdS/CFT prescription which maps to the boundary all the information contained in the bulk. In particular, we show that the divergent local terms of the on-shell action contain information about the Legendre transformed generating functional, and that the new constraints for which the irregular modes propagate in the bulk are the same constraints for which such divergent local terms cancel out. In this situation, the addition of counterterms is not required. We also show that there exist particular cases for which the unitarity bound is reached, and the conformal dimension becomes independent of the effective mass. This phenomenon has no bulk counterpart.
\end{abstract}

PACS numbers: 11.10.Kk 04.62.+v

Keywords: AdS/CFT Correspondence, Energy, Boundary Conditions

\footnotetext{
${ }^{1}$ pablo@fma.if.usp.br

${ }^{2}$ rivelles@fma.if.usp.br
} 


\section{Introduction}

Since the proposal of Maldacena conjecturing the existence of a duality between Type IIB supergravity theory on Anti-de Sitter (AdS) space and the large N limit of a conformal $\mathcal{N}=4$ Super Yang-Mills theory living on the boundary of the AdS space [四], a large amount of work has been devoted to understand all the implications of the so-called AdS/CFT correspondence. For the $\mathrm{d}+1$ dimensional AdS space $\left(A d S_{d+1}\right)$, the explicit prescription for mapping one theory into the other has been given in [2] [3], and it takes the form

$$
Z_{A d S}\left[\phi_{0}\right]=\int_{\phi_{0}} \mathcal{D} \phi \exp (-I[\phi]) \equiv Z_{C F T}\left[\phi_{0}\right]=\left\langle\exp \left(\int_{\partial \Omega} d^{d} x \mathcal{O} \phi_{0}\right)\right\rangle,
$$

where $\phi_{o}$ is the boundary value of the bulk field $\phi$ which couples to the boundary conformal field theory $(\mathrm{CFT})$ operator $\mathcal{O}$. In this prescription, the path integral over the bulk fields on the l.h.s is computed under the restriction that the field $\phi$ satisfies a Dirichlet boundary condition on the boundary of AdS. The above Dirichlet prescription has been successfully applied to the cases of the scalar field [2] [4] [5] [6] [7], the spinor field [8] [9] [10], [1], the vector field [2] [4] [9] [12] [13, the Rarita-Schwinger field [14] [15] [16], the graviton field [17] [18], the massive symmetric tensor field [19] and the antisymmetric $p$-form field [20][21]. In all cases, the boundary CFT two-point and higher order functions obtained through the mapping Eq.(1) were shown to have the form prescribed by conformal invariance, and the conformal dimensions of the boundary operators corresponding to all the fields listed above were established. We remark that, in all references above but [7], only Dirichlet boundary conditions were employed. Further refinements include the addition of local counterterms to the action

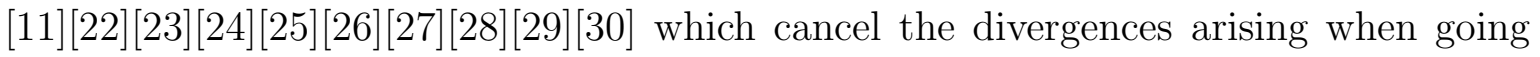
to the boundary. We also mention the work on holographic renormalization group flows from deformations on AdS which extends the AdS/CFT correspondence to nonconformal field theories 31 32 33 34. 34.

In this work, we concentrate on the formulation of the scalar field theory on AdS spaces in both minimally and non-minimally coupled cases. It is known [35] 36] that the usual quantization of the scalar field theory on AdS spaces in global coordinates' involves two different kinds of normalizable modes, namely the 'regular' and 'irregular' ones. Since there are two possible consistent quantizations of the scalar field on AdS, we expect that for each one of them there exists a corresponding boundary CFT. But,

\footnotetext{
${ }^{3}$ For recent work on quantization in Poincaré coordinates, see [37] 38] [39]
} 
as pointed out in [23], the Dirichlet prescription Eq.(11) accounts only for the CFT that corresponds to the situation in which regular modes propagate in the bulk. In order to also account for the missing CFT, the proposal in [23] is that its generating functional can be obtained by Legendre transforming the original one which corresponds to regular modes. It has been shown in [23] that, in fact, this procedure gives rise to a boundary CFT whose conformal dimension is the one expected for irregular modes propagating in the bulk.

Then, at first sight, it seems that the usual quantization of the scalar field theory on AdS along the lines of [35] 36] [40], together with the AdS/CFT formulation which makes use of the Dirichlet prescription Eq.(1) and the Legendre transform prescription in [23], gives rise to a consistent formulation of the scalar field theory on AdS spaces and in the AdS/CFT correspondence. However, we will show in this article that the usual quantization in global coordinates, as developed in [35] 36] 40], imposes constraints on the mass and the coupling coefficient of the field to the metric which cannot be mapped to the boundary through the usual Dirichlet and Legendre transform prescriptions. It is a serious drawback, because the very first thing that we require on any AdS/CFT prescription is that it must be able to map to the boundary all the information contained in the AdS bulk. Then, in order to remove these difficulties, we will argue that the usual energy, which is constructed through the 'improved' stress-energy tensor, as in [35] 36] 40], is not the natural one to be considered in the AdS/CFT correspondence context. This will require a new definition of energy, associated to the Noether current corresponding to time displacements, which is the natural one to be considered in the context of the AdS/CFT correspondence. As a result, we will perform, for both the minimally and non-minimally coupled cases, a new consistent quantization of the scalar field theory on AdS in global coordinates. We will show that this definition of energy is sensitive to the boundary conditions. Thus, we will compute it for all possible boundary conditions on the scalar field, namely Dirichlet, Neumann, and a combination of both of them which we call mixed boundary condition, but that sometimes is also called as Robin or third boundary condition. We will show that the energy is conserved, positive and finite for regular and irregular modes arising for some constraints on the mass and the coefficients of mixed boundary conditions thus leading to a consistent quantization of the scalar field theory on the AdS bulk in global coordinates. Besides, in this new picture the Breitenlohner-Freedman bound [35] 36] 40] still holds, as expected.

Then, in the AdS/CFT context, we will look for generalizations of the usual Dirichlet and Legendre transform prescriptions for which the boundary CFT's contain all the information about the new constraints just mentioned and the regular and irregular modes propagating in the bulk. We will show that the incorporation of boundary 
conditions leads, in a natural way, to a generalized AdS/CFT prescription in which conformal operators couple at the boundary to sources which depend on the selected boundary condition.

We will also consider a generalized prescription for the Legendre transform, in which we transform the whole action at the boundary, rather than only the leading non-local term, as in the usual prescription. This generalized prescription makes use, in fact, of the standard form of the Legendre transformation. At first sight, it could seem that such a generalization of the usual prescription introduces no new results. However, this is not the case, because, as we will show, the divergent local terms of the on-shell action contain information about the Legendre transformed generating functional, and then, the operations of expanding in powers of the distance to the boundary and then selecting the generating functional, and of performing the Legendre transformation, are not commuting operations. As a consequence, we will show that the generalized prescription gives rise to results which, in general, are different from those arising from the usual Legendre transform prescription. A key result will be that the new results arising from the generalized AdS/CFT prescription are in whole agreement with the ones that we find on performing the quantization in the bulk. In addition, this generalized Legendre transform prescription will let us to remove some difficulties that appear in the usual prescription.

In general, we will show that our formulation gives rise to boundary CFT's which contain all the information about the quantization of the scalar field theory on AdS in global coordinates, and, in particular, we will also show that the constraints for which the irregular modes propagate in the bulk are the same constraints for which the divergent local terms of the on-shell action cancel out. In this situation, the addition of counterterms is not required. We will also show that there exists one particular case which has no bulk counterpart, namely the one in which the conformal dimension reaches the unitarity bound and becomes independent of the effective mass.

The paper is organized as follows. In Section 2, we motivate the introduction of a new formalism for the scalar field theory on AdS and in the AdS/CFT correspondence. We consider some difficulties regarding the usual formulation, and provide some clues for a new formalism in which such difficulties can be removed. In Section 3, we compute the stationary actions corresponding to Dirichlet, Neumann and mixed boundary conditions on the scalar field in both the minimally and non-minimally coupled cases, and then, for each action, we construct the Noether current corresponding to time displacements. We show that it is sensitive to the addition of boundary terms to the action, and that it is conserved, in the sense that its covariant divergence vanishes. Then, we compute the energy associated to the Noether current, and find the con- 
straints for which it is conserved, positive and finite. In this way, we can consistently quantize the scalar field theory on AdS. In particular, we compute the new constraints for which the irregular modes propagate in the bulk. In Section 4, we consider the generalized AdS/CFT prescription, and show that it leads to results which are in complete agreement with those obtained by performing the quantization in global coordinates. In particular, we show that the new constraints for which irregular modes propagate in the bulk are reproduced, in a natural way, by the generalized AdS/CFT prescription. This is a remarkable non-trivial result which can be considered as a strong evidence in support of our proposed formalism. We will also show that the divergent local terms of the on-shell action contain information about the Legendre transformed generating functionals, and that the new constraints for which the irregular modes propagate in the bulk are the same for which such divergent local terms cancel out, so that no counterterms are required. Finally, Section 5 presents our conclusions. Appendix A contains some useful identities for the hypergeometric functions, and Appendix B presents the main results obtained from the generalized AdS/CFT prescription.

\section{Motivations}

In order to set up the notation and motivate the subsequent development, we begin this section by reviewing the main results of the usual formulation of scalar field theory on AdS spaces and in the AdS/CFT correspondence. Most of the results presented in this section will be used throughout this paper.

A consistent quantization of a massive real scalar field theory on $A d S_{4}$ was found in [35] 36] (see also [41] for earlier results) and then extended to $A d S_{d+1}$ in [40]. It will be useful to begin by concentrating on the minimally coupled case. Consider the usual action in $A d S_{d+1}$

$$
-\frac{1}{2} \int d^{d+1} x \sqrt{g}\left(g^{\mu \nu} \partial_{\mu} \phi \partial_{\nu} \phi+m^{2} \phi^{2}\right)
$$

where $m$ is the mass of the scalar field, and $g_{\mu \nu}$ is a metric of 'mostly plus' signature describing $A d S_{d+1}$. In the formulation of [35 36] 441, the solution of the classical equation of motion of the scalar field $\left(\nabla^{2}-m^{2}\right) \phi=0$ is expanded in modes

$$
\phi=\sum_{n}\left[a_{n} \phi_{n}+a_{n}^{*} \phi_{n}^{*}\right]
$$

for which the following scalar product is defined 


$$
\left(\phi_{n}, \phi_{n^{\prime}}\right)=i \int d^{d} x \sqrt{g} g^{0 \nu}\left[\phi_{n} \partial_{\nu} \phi_{n^{\prime}}^{*}-\partial_{\nu} \phi_{n} \phi_{n^{\prime}}^{*}\right] .
$$

Here, the $a_{n}$ 's are arbitrary complex coefficients, and $n$ labels the modes. The above scalar product is the 'conserved' charge corresponding to the conserved current (in the sense that its covariant divergence vanishes)

$$
H^{\mu}=i g^{\mu \nu}\left[\phi_{n} \partial_{\nu} \phi_{n^{\prime}}^{*}-\partial_{\nu} \phi_{n} \phi_{n^{\prime}}^{*}\right] .
$$

The problem which arises when quantizing the scalar field theory on AdS is that this space does not admit a Cauchy surface, and a massless particle reaches the spatial infinity in a finite time. The solution proposed in [35] 36] 441] for performing a consistent quantization was to compactify the space and impose appropriate boundary conditions on the solution of the classical equation of motion, such as the scalar product Eq.(任) be actually conserved. This means to require that there is no flux of the current Eq.(5) through the boundary at spatial infinite. This imposes further conditions on the solution of the equation of motion, and the result in 35] 36] 40] is that there exist two different asymptotic behaviors of the scalar field such that the flux at the boundary vanishes. They are called 'regular' and 'irregular' modes, and they behave close to the boundary as

$$
\phi_{R} \sim \hat{\epsilon}^{\Delta_{+}^{(0)}}, \quad \phi_{I} \sim \hat{\epsilon}^{\Delta_{-}^{(0)}}
$$

where $\phi_{R}, \phi_{I}$ correspond to regular and irregular modes respectively, $\hat{\epsilon}$ is a measure of the distance to the boundary which is considered to be small, and

$$
\begin{gathered}
\Delta_{ \pm}^{(0)}=\frac{d}{2} \pm \nu^{(0)}, \\
\nu^{(0)}=\sqrt{\frac{d^{2}}{4}+m^{2}} .
\end{gathered}
$$

One important result in 35] 36] is that, whereas for regular modes the flux of the current Eq.(5) vanishes for any $\nu^{(0)} \geq 0$, for the irregular ones it vanishes only for

$$
0 \leq \nu^{(0)}<1
$$

Since regular and irregular modes are normalizable, the scalar field can be consistently quantized. Note that for $\nu^{(0)}>1$ there is only one possible quantization, namely the one corresponding to regular modes. On the other hand, for $0 \leq \nu^{(0)}<1$ both 
regular and irregular modes are normalizable, and then we have two possible consistent quantizations. In spite of the fact that the regular solution has been much more studied than the irregular one, both of them exist and lead to consistent formulations. It is also important to point out that stability imposes the condition that $\nu^{(0)}$ is a real number [35] 36]. Such a condition can be written as

$$
m^{2} \geq-\frac{d^{2}}{4}
$$

which is known as the Breitenlohner-Freedman bound.

The analysis of regular and irregular modes in the context of the AdS/CFT correspondence was performed in [6], where it has been suggested that there are two kinds of modes which are relevant to the formulation of the scalar field theory on AdS, namely the quantum, fluctuating modes which propagate in the bulk, and the classical, nonfluctuating backgrounds which couple to the boundary conformal operators through the AdS/CFT prescription Eq.(11). For $\nu^{(0)}>1$, only regular modes are normalizable, and thus they are the only kind of modes which propagate in the bulk. In this situation, the irregular modes are the classical, non-fluctuating backgrounds which couple to the boundary conformal operator, that happens to have conformal dimension $\Delta_{+}^{(0)}$. This result has been successfully reproduced through the Dirichlet prescription Eq.(1) [2] [4].

However, a new feature arises for $0 \leq \nu^{(0)}<1$. In this situation, both regular and irregular modes are normalizable, and thus we have two possible consistent quantizations. Since the AdS/CFT correspondence states that any quantum field theory on AdS is equivalent to a conformal theory on the boundary, we expect that we should have two different boundary CFT's. One of them corresponds to the situation in which we choose the regular modes to describe the quantum fluctuations propagating in the bulk. In this case, we expect to have a boundary conformal operator of conformal dimension $\Delta_{+}^{(0)}$, and this CFT can be reproduced through the Dirichlet prescription Eq.(1), as it happens for $\nu^{(0)}>1$. The problem arises when we choose the irregular modes to describe the quantum fluctuations propagating in the bulk. In this situation, we expect the boundary conformal operator to have conformal dimension $\Delta_{-}^{(0)}$. However, as pointed out in [23], such an operator cannot be reproduced through the Dirichlet prescription Eq.(11). It is also important to note that the conformal dimension $\Delta_{+}^{(0)}$ is bounded from below by $d / 2$, whereas the constraint Eq.(9) makes the conformal dimension $\Delta_{-}^{(0)}$ to be bounded from below by $(d-2) / 2$, which is precisely the unitarity bound for scalar operators in $d$ dimensional field theory. The bound $d / 2$ is more stringent than the unitarity bound $(d-2) / 2$, and in fact there exist particular examples 
for which the conformal dimension $\Delta_{-}^{(0)}$ is needed (for details, see [23] and references therein).

From all considerations above, we conclude that, in order to be able to reproduce not only the conformal dimension $\Delta_{+}^{(0)}$ but also the missing conformal dimension $\Delta_{-}^{(0)}$, some generalization of the Dirichlet prescription Eq.(四) must be introduced. In doing so, the proposal in [23] is that the generating functional in the theory with conformal dimension $\Delta_{-}^{(0)}$ is a Legendre transform of the generating functional in the theory with conformal dimension $\Delta_{+}^{(0)}$ (see also 42 for previous results arising from group-theoretic analysis). It is known [2] [4] [5] that such a generating functional is written in momentum space as

$$
S\left[\phi_{0}\right]=\frac{\Gamma(1-\nu)}{\Gamma(\nu)} \int \frac{d^{d} k}{(2 \pi)^{d}} \phi_{0}(\vec{k}) \phi_{0}(-\vec{k})\left(\frac{k}{2}\right)^{2 \nu},
$$

where $k=|\vec{k}|$. The prescription in [23] is to carry out the Legendre transformation by setting

$$
\tilde{S}\left[\phi_{0}, \tilde{\phi}_{0}\right]=S\left[\phi_{0}\right]+\alpha \int \frac{d^{d} k}{(2 \pi)^{d}} \phi_{0}(\vec{k}) \tilde{\phi}_{0}(-\vec{k})
$$

where the coefficient $\alpha$ has been chosen in [23] to be $\alpha=2 \Delta^{(0)}-d$. The generating functional in the theory with conformal dimension $\Delta_{-}^{(0)}$ is the minimum of $\tilde{S}\left[\phi_{0}, \tilde{\phi}_{0}\right]$ with respect to $\phi_{0}$ (for fixed $\tilde{\phi}_{0}$ ), and it is given by

$$
\tilde{S}\left[\tilde{\phi}_{0}\right]=-\frac{\alpha^{2}}{4} \frac{\Gamma(\nu)}{\Gamma(1-\nu)} \int \frac{d^{d} k}{(2 \pi)^{d}} \tilde{\phi}_{0}(\vec{k}) \tilde{\phi}_{0}(-\vec{k})\left(\frac{k}{2}\right)^{-2 \nu} .
$$

Integration over the momentum shows that the field $\tilde{\phi}_{0}$ couples to a boundary conformal operator $\tilde{\mathcal{O}}$ of conformal dimension $\Delta_{-}^{(0)}$ through the prescription 23]

$$
\exp (-\tilde{S}) \equiv\left\langle\exp \left(\int d^{d} x \tilde{\mathcal{O}}(\vec{x}) \tilde{\phi}_{0}(\vec{x})\right)\right\rangle
$$

which arises from the prescription Eq.(1) by considering the transformed field $\tilde{\phi}_{0}$ as the source for the new boundary conformal operator $\tilde{\mathcal{O}}$.

Thus, at first sight, it seems that the AdS/CFT prescription Eqs.(11, 12) successfully maps to the boundary all the information obtained in [35] 36] 40] on performing the quantization of the scalar field theory on the AdS bulk. However, one of the purposes of this work is to point out that there remain some open problems which need to be 
considered. One of them is the fact that the constraint Eq.(1) for which the irregular modes are normalizable has not so far been reproduced by any AdS/CFT prescription. This is so because the Legendre transformation Eq.(12) can, in principle, be carried out for any value of $\nu$. Thus, in the usual prescription, the constraint Eq.(91) has to be imposed 'by hand'. This is a serious drawback, because the very first thing that we require on any AdS/CFT prescription is that it must be able to map to the boundary all the information contained in the bulk. In this particular case, we would expect that the AdS/CFT prescription could reproduce the constraint Eq.(9) in a natural way, just as the analysis along the lines of [35] [36] does in the bulk.

There is still another problem regarding the usual Dirichlet and Legendre transform prescriptions Eqs.(1, 12), related to the existence of a further constraint which they cannot reproduce, and that needs to be imposed 'by hand' too. Such a constraint arises on considering the role of the energy in the usual quantization of the scalar field theory on AdS, along the lines of [35] [36] [40]. In order to make this clear, we begin by considering, instead of Eq.(2), a new action in which the scalar field is now non-minimally coupled to the $A d S_{d+1}$ metric

$$
I_{\text {matter }}=-\frac{1}{2} \int d^{d+1} x \sqrt{g}\left[g^{\mu \nu} \partial_{\mu} \phi \partial_{\nu} \phi+\left(m^{2}+\varrho R\right) \phi^{2}\right],
$$

where $R$ is the Ricci scalar corresponding to $A d S_{d+1}$ (note that it is a constant), and $\varrho$ is an arbitrary coupling coefficient. In this situation, the non-minimal coupling introduces a new effective mass

$$
M^{2}(\varrho)=m^{2}+\varrho R,
$$

and now the regular and irregular modes for which the current Eq.(司) is conserved behave, close to the boundary, as follows

$$
\phi_{R} \sim \hat{\epsilon}^{\Delta_{+}(\varrho)}, \quad \phi_{I} \sim \hat{\epsilon}^{\Delta_{-}(\varrho)},
$$

where

$$
\begin{gathered}
\Delta_{ \pm}(\varrho)=\frac{d}{2} \pm \nu(\varrho), \\
\nu(\varrho)=\sqrt{\frac{d^{2}}{4}+M^{2}(\varrho)} .
\end{gathered}
$$


In particular, the coefficients $\Delta_{ \pm}^{(0)}$ and $\nu^{(0)}$ in Eqs. (7, 8) are just the coefficients $\Delta_{ \pm}(0)$ and $\nu(0)$ respectively, and the constraint Eq.(9) for irregular modes is generalized as follows

$$
0 \leq \nu(\varrho)<1 .
$$

Note that the Breitenlohner-Freedman bound now reads

$$
M^{2}(\varrho) \geq-\frac{d^{2}}{4},
$$

instead of Eq.(10).

Under an infinitesimal variation of the metric

$$
g_{\mu \nu} \rightarrow g_{\mu \nu}+\delta g_{\mu \nu}
$$

the action Eq.(15) transforms as

$$
\delta_{g} I_{\text {matter }}=-\frac{1}{2} \int d^{d+1} x \sqrt{g} T_{\mu \nu} \delta g^{\mu \nu}+\text { surface terms, }
$$

where $T_{\mu \nu}$ is the 'improved' stress-energy tensor of the scalar field. It is given by

$$
T_{\mu \nu}=\partial_{\mu} \phi \partial_{\nu} \phi-\frac{1}{2} g_{\mu \nu}\left[g^{\alpha \beta} \partial_{\alpha} \phi \partial_{\beta} \phi+M^{2}(\varrho) \phi^{2}\right]+\varrho\left(g_{\mu \nu} \nabla^{2}-\nabla_{\mu} \nabla_{\nu}+R_{\mu \nu}\right) \phi^{2},
$$

where $R_{\mu \nu}$ is the Ricci tensor of $A d S_{d+1}$ and $\nabla_{\mu}$ stands for a covariant derivative. The analysis in [35] [36] is based on a previous formulation in [41] 43] and, in particular, it defines the contribution of the scalar field to the energy of the system as the 'conserved' charge corresponding to the conserved current

$$
J^{\mu}=-T_{0}^{\mu},
$$

where we have contracted the stress-energy tensor with the Killing vector corresponding to time displacements in global coordinates. Note that we have included a minus sign because of the 'mostly plus' signature of the metric. For future purposes, it will be useful to call the 'conserved' quantity described above as the 'metrical energy'. In order for the 'conserved' metrical energy to be actually conserved, Breitenlohner and Freedman impose that there is no flux of the current Eq.(25) through the boundary (see also [40] for the extension to the $\mathrm{d}+1$ dimensional case), and this restriction fixes further conditions on the solution of the equation of motion. The result is that, whereas for 
regular modes the metrical energy is actually conserved for any value of $\varrho$, for irregular modes it is conserved only for $\varrho$ being a solution of the constraint

$$
\varrho=\frac{1}{2} \frac{\Delta_{-}(\varrho)}{1+2 \Delta_{-}(\varrho)} .
$$

This new constraint must be added to the usual one Eq.(20) that we have found on considering the conservation of the scalar product Eq.(4) for irregular modes.

A very important result in [35] 36] 40] is that for regular modes and irregular ones satisfying Eqs.(20, 26), and only for them, the metrical energy is not only conserved, but also positive and finite. This leads to a consistent quantization of the scalar field theory on AdS in the non-minimally coupled case. In particular, for $0 \leq \nu<1$ we have again two possible quantizations corresponding to the situations in which the modes which propagate in the bulk are chosen to be the regular or the irregular ones. On the other hand, for $\nu>1$ we have only one possible consistent quantization, namely the one in which the regular modes propagate in the bulk.

Now we are ready to explain the second problem regarding the usual Dirichlet and Legendre transform prescriptions Eqs.(11, 12). Note that in such formulation, the coupling between the scalar field and the metric is understood just as a mere renormalization of the mass (see Eq.(16)). In particular, the Legendre transformation Eq.(12) can be performed for any value of $\varrho$, and thus, in this formalism, the constraint Eq.(26) cannot be reproduced, just as it happens to the constraint Eq.(20) as pointed out before. Again, we are facing a serious drawback.

Summarizing, the Legendre transform prescription Eq.(12) is able to reproduce the missing conformal dimension $\Delta_{-}(\varrho)$, but it has no information about the constraints on $\nu$ and $\varrho$ for which irregular modes propagate in the bulk.

Furthermore, there still remain two more problems regarding the usual Legendre transform prescription. One of them is that it does not fix the coefficient $\alpha$ in Eq.(12). In [23], it has been chosen to be $\alpha=2 \Delta-d$ under the conjecture that the conjugated field of $\phi_{0}$ is actually $(2 \Delta-d) \tilde{\phi}_{0}$ rather than $\tilde{\phi}_{0}$.f We are looking for a prescription which fixes $\alpha$ in a natural way, thus leaving no coefficient to be chosen 'by hand'. This means that, instead of including $\alpha$ in the Legendre transform prescription, we want the Legendre transformation itself to fix the precise form of the conjugated field.

And the final problem regarding the usual AdS/CFT prescription is related to the

\footnotetext{
${ }^{4}$ The calculation in 23] involves taking the derivative of the renormalized on-shell action and compute the expectation value of the dual operator to the field. Then, after having computed the coefficient $\alpha$, it is included in the Legendre transform prescription.
} 
fact that for integer $\nu$ the on-shell action also contains logarithmic terms which are not present for non-integer $\nu$. For instance, for $\nu=0$ the generating functional is written in momentum space as [7]

$$
S\left[\phi_{0}\right]=\frac{1}{2} \int \frac{d^{d} k}{(2 \pi)^{d}} \phi_{0}(\vec{k}) \phi_{0}(-\vec{k}) \ln k,
$$

instead of Eq.(11). The case $\nu=0$ is included in the interval Eq.(20) for which irregular modes propagate in the bulk. In fact, this is the particular case for which the conformal dimensions $\Delta_{+}$and $\Delta_{-}$are equal. Thus, for $\nu=0$ we would expect the generating functional to be self-conjugated. However, it is easy to verify that, in this case, the usual Legendre transform prescription Eq.(12) does not work, due to the presence of the logarithmic term in Eq.(27). This indicates that something is being missed in the usual formulation.

For all these reasons, we will propose a new formulation of the scalar field theory on AdS and in the AdS/CFT correspondence, in both minimally and non-minimally coupled cases, for which all the difficulties that arise from the usual formulation can be removed. In order to do this, we begin by concentrating on the definition of the energy of the scalar field theory on the AdS bulk. Note that the AdS/CFT correspondence is sensitive to the addition of boundary terms to the action. This can be seen to be true by computing the 1.h.s of Eq.(1) for a classical field configuration. All that is left is a boundary term. If we start with different boundary terms in the action then we obtain different correlation functions on the r.h.s. But if the AdS/CFT correspondence is sensitive to the addition of boundary terms, and we require that it must also contain all the information regarding the conservation, positivity and finiteness conditions on the energy of the theory on the bulk, then it is natural to demand that the energy of the theory on the bulk has also to be sensitive to the addition of boundary terms to the action. However, the metrical energy, which is the one that is analyzed in the usual formulation along the lines of [35] 36] 40], does not fulfil this requirement. This is so because, as it can easily be verified, the addition of a boundary term to the action Eq.(15) amounts to the addition of only new surface terms in Eq.(23), and it means that the stress-energy tensor Eq.(24) is not sensitive to the addition of boundary terms to the action.

The analysis above leads us to consider the possibility that, in fact, the metrical

\footnotetext{
${ }^{5}$ One could argue that the surface terms in Eq.(23) contribute to the stress-energy tensor with terms containing delta functions. However, such surface terms vanish, because we always impose proper boundary conditions. This topic will be discussed in detail in Section 3 .
} 
energy is not the natural definition of the energy in the AdS/CFT correspondence context. Thus, we are forced to look for a new definition of the energy, and a brief analysis shows that the natural candidate is the 'conserved' charge which is constructed out of the Noether current corresponding to time displacements. This is so because the new 'conserved' charge, which from now on we call the 'canonical energy', is sensitive to the addition of boundary terms to the action, a property inherited from the Noether currents.

The choice of the canonical energy to describe the energy of the bulk theory may seem strange because, in general, only the metrical energy is employed. This happens because the stress-energy tensor Eq.(24) has some nice properties, namely, that it is symmetric and it is also traceless for Weyl-invariant theories (the critical coupling coefficient is $\left.\varrho=\frac{d-1}{4 d}\right)$. However, in principle, we could find physical systems for which the canonical energy acquires relevance. As an example of this, we mention black holes physics, in whose context some aspects of the relation between the metrical and canonical energies have been analyzed in [44. There, it has been established that both energies are related to different physical properties of the system. In the case of the metrical energy, it was shown that it is the physical energy which enters the first law of black hole thermodynamics. In the case of the canonical energy, the result in 44 is that it is the generator of the time evolution. This is the reason why the author of [44 calls the metrical and canonical energies as the 'energy' and the 'Hamiltonian', respectively. Here, we will not attempt to perform, for the case of AdS spaces, a formal demonstration of the statement that the canonical energy is the generator of the time evolution. We just mention that, on computing the Poisson brackets, the presence of a boundary requires the addition of some surface terms to assure that the Jacobi identity is satisfied 445] [46].

One of the purposes of this paper is to present some evidence in support of our conjecture that the canonical energy is the natural definition of the energy in the AdS/CFT correspondence context. In order to do this, we start by constructing the canonical energy. We know that it is sensitive to the addition of boundary terms to the action. The origin of boundary terms in the action is due to the variational principle. In order to have a stationary action boundary terms, which will depend on the choice of the boundary conditions, must be introduced. An example of this is the Gibbons-Hawking term [47] that is added to the Einstein-Hilbert action in order to make it stationary when the metric is fixed at the boundary. Other examples can be found in the AdS/CFT correspondence context, where the boundary terms that make the action stationary have been shown to be the generating functionals of the boundary CFT's for the cases of the spinor field [10] [11] and the Self-Dual model [12]. 
But, if as a first step, we need to construct the canonical energy, and it depends on the boundary conditions, then we must consider all possible boundary conditions in order to compute all possible canonical energies of the system. This leads us to analyze Dirichlet, Neumann and mixed boundary conditions on the bulk scalar field. As we will show, an interesting feature about mixed boundary conditions is that they form a one-parameter family of boundary conditions. Thus, there is an infinite number of mixed boundary conditions. In this article, we will develop a new formalism in which we start from the usual actions for both the minimally and non-minimally coupled scalar fields (see Eqs.(2), 15)). Then, we will add surface terms to such actions in order to make them stationary under Dirichlet, Neumann and mixed boundary conditions on the scalar field. On doing this, we will also keep an eye on the requirement that the actions must also be stationary under variations of the metric which vanish at the border. In particular, for mixed boundary conditions we will find a one-parameter family of surface terms.

Then, for each resulting action, we will compute the Noether current corresponding to time displacements in global coordinates and show that it is conserved, in the sense that its covariant divergence vanishes. In the particular case of mixed boundary conditions, there will be real parameters labelling the corresponding surface terms, and we will show that there is a deep analogy between the way in which the coupling coefficient $\varrho$ in Eq.(15) is related to the addition of an 'improvement' term to the stress-energy tensor (see Eq.(24)), and the way in which the parameters of mixed boundary conditions are related to the addition of new 'improvement' terms to the Noether current. Note that, in general, the Noether current will be different from the usual choice of Eq.(25). It will give rise to a 'conserved' charge which is the canonical energy that we have discussed before. Then, we will perform a consistent quantization of the scalar field theory on AdS spaces in global coordinates. This quantization is, in many respects, analogous to the one performed in [35] 36] 40]. The difference is that, in this case, we will carry out the calculations making use of the canonical energy instead of the metrical one. In particular, we will find again regular and irregular modes for which the canonical energy is conserved, and a key result will be that for such solutions, and only for them, the canonical energy is also positive and finite. This will lead us to obtain a new consistent quantization of the scalar field theory on AdS. We remark that the Breitenlohner-Freedman bound Eq.(21), together with the constraint Eq.(20) for which irregular modes propagate, still hold in this formalism. We will show that the important difference between the quantizations which make use of the metrical and canonical energies is that the constraint Eq.(26), for which the metrical energy is conserved, positive and finite for irregular modes propagating in the bulk, is replaced 
by new constraints to be found later. A key result that we will also show is that, unlike the usual constraint Eq.(26), these new constraints can be reproduced by means of a generalized version of the AdS/CFT prescription Eqs.(11, 12) which we will introduce below.

Once we have performed the quantization of the scalar field theory on AdS in global coordinates, the next step will be to establish whether the new results can be mapped through the AdS/CFT correspondence. An important observation to be made is that the Dirichlet, Neumann and mixed boundary conditions which are considered on analyzing Noether currents in global coordinates play a fundamental role in our generalized AdS/CFT prescription, because they fix, in a natural way, the source which couples to the boundary conformal operators. It means that, instead of the usual Dirichlet prescription

$$
\exp \left(-I_{A d S}\left[\phi_{0}\right]\right) \equiv\left\langle\exp \left(\int d^{d} x \mathcal{O}(\vec{x}) \phi_{0}(\vec{x})\right)\right\rangle
$$

the considerations above lead us to employ a generalized AdS/CFT prescription of the form

$$
\exp \left(-I_{A d S}\left[A_{0}\right]\right) \equiv\left\langle\exp \left(\int d^{d} x \mathcal{O}(\vec{x}) A_{0}(\vec{x})\right)\right\rangle
$$

where the source $A_{0}$ which couples to the boundary conformal operator depends on the boundary conditions. It means that for Dirichlet, Neumann and mixed boundary conditions the source $A_{0}$ will be the field, its Lie derivative along the normal vector to the boundary, or a combination of both of them, respectively, computed at the border. Thus, the concept of canonical energy that we considered on performing the quantization of the scalar field leads us, in a natural way, to a generalization of the usual Dirichlet prescription Eq.(28). The analysis of this generalized prescription has been carried out in [7] for the particular minimally coupled case, and we show in this paper that the root of such a generalized formalism can be found on the concept of canonical energy. We stress the fact that, in considering the non-minimally coupled case, we will find new results which have not been considered in [7].

A final analysis that we will perform in this article regards a generalized Legendre transform prescription which does not present the difficulties mentioned earlier. We also aim to integrate such a Legendre transform into the generalized prescription Eq.(29) in such a way that there exists an exact agreement between the resulting AdS/CFT prescription and the quantization that we develop in this paper. 
Note that the usual Legendre transform prescription Eq. (12) involves a procedure in which one first expands the on-shell action in powers of the distance to the boundary, then selects the leading non-local term, i.e. the generating functional Eq.(11), and only then performs the Legendre transformation Eq.(12). This means that, in the usual formulation along the lines of [23], from all local and non-local terms of the on-shell action only the leading non-local term is taken into account to perform the Legendre transformation. At first sight, it may seem natural to do so, since the non-leading terms vanish when the action is taken to the boundary, and the divergent local terms are of no relevance on computing the non-local part of the boundary CFT two-point function. However, we claim in this work that, even when local terms do not need to be taken into account in computing the original generating functional, they, in general, contain information about the Legendre transformed one, and thus they have to be taken into account when performing the Legendre transformation. In other words, the key observation to be made is that the operations of expanding the on-shell action in powers of the distance to the boundary and then select the leading non-local term, and of performing the Legendre transformation, are not commuting operations. This means that we have to consider a new prescription in which we start by performing the Legendre transformation on the whole on-shell action, and only then we select the transformed generating functional, unlike the usual prescription in [23] in which the inverse procedure is considered. We will show that, in general, this generalized prescription gives rise to results which are different from those in 23], but that are in complete agreement with the results arising from the new quantization of the scalar field theory on the AdS bulk. We will also show that the constraints for which the irregular modes propagate in the bulk are the same constraints for which the divergent local terms of the on-shell action vanish. In this situation, the addition of counterterms is not required.

The generalized prescription for the Legendre transform makes use, in fact, of the standard form of the Legendre transformation, and it can be written schematically as

$$
\tilde{I}_{A d S}\left[A_{0}, \tilde{A}_{0}\right]=I_{A d S}\left[A_{0}\right]-\int \frac{d^{d} k}{(2 \pi)^{d}} A_{0}(\vec{k}) \tilde{A}_{0}(-\vec{k}) .
$$

Note that, unlike the usual Legendre transform prescription Eq.(12), where the Legendre transformation acts only on the leading non-local term $S\left[\phi_{0}\right]$, in the Legendre transformation above we transform the whole on-shell action $I_{A d S}\left[A_{0}\right]$. We stress the fact that, in general, this Legendre transformation gives rise to generating functionals of the boundary CFT's which are different from the one that is obtained through the 
usual Legendre transform prescription Eq.(12). The Legendre transform of $I_{A d S}\left[A_{0}\right]$ is given by the minimum of $\tilde{I}_{A d S}\left[A_{0}, \tilde{A}_{0}\right]$ with respect to $A_{0}$ (for fixed $\tilde{A}_{0}$ ). An important observation is that the Legendre transformation Eq.(30) not only modifies the usual Legendre transform prescription by acting on the whole on-shell action, but also acts on the much more general source $A_{0}$, as required before. The inclusion of the generic source $A_{0}$ realizes, in a natural way, the integration of the Legendre transformation Eq.(30) into the generalized AdS/CFT prescription Eq.(29), whose corresponding 'conjugated' prescription reads

$$
\exp \left(-\tilde{I}_{A d S}\left[\tilde{A}_{0}\right]\right) \equiv\left\langle\exp \left(\int d^{d} x \tilde{\mathcal{O}}(\vec{x}) \tilde{A}_{0}(\vec{x})\right)\right\rangle,
$$

where $\tilde{\mathcal{O}}$ is the new conformal operator which couples to the Legendre transformed source $\tilde{A}_{0}$. Note that, for each boundary condition, there will be two generating functionals, namely those arising from the generalized prescriptions Eqs.(29, 31). In both cases, we will also have additional local and non-local terms. We remark that the Legendre transformation Eq.(30) does not impose any constraints on $\nu$ or $\varrho$. The role of the Legendre transformation is to interpolate between the boundary CFT's corresponding to regular and irregular modes propagating in the bulk. On the other hand, we will show that the constraints for which irregular modes propagate in the bulk are fixed, in a natural way, by the generalized AdS/CFT prescription Eqs.(29, 31), and thus they are not fixed 'by hand', as it happens in the usual formalism. In the cases for which only regular modes propagate in the bulk, we will show that the Legendre transformation Eq.(30) interpolates between self-conjugated theories with conformal dimension $\Delta_{+}(\varrho)$, as expected. On the other hand, for the cases for which the irregular modes can propagate in the bulk too, we will show that the Legendre transformation Eq.(30) interpolates between the conformal dimensions $\Delta_{+}(\varrho)$ and $\Delta_{-}(\varrho)$. In some cases, the conformal dimension $\Delta_{+}(\varrho)$ belongs to the original generating functional, and then the conformal dimension $\Delta_{-}(\varrho)$ arises from the corresponding Legendre transformed generating functional. However, another new property of this generalized formalism is that, as we will show, there also exist cases for which it is the conformal dimension $\Delta_{-}(\varrho)$, instead of the usual one $\Delta_{+}(\varrho)$, that arises from the original generating functional, and then the conformal dimension $\Delta_{+}(\varrho)$ is obtained from the Legendre transformed generating functional, as expected. It is important to stress that the cases for which the Legendre transformation Eq.(30) interpolates between different conformal dimensions are precisely the same for which the divergent local terms of the on-shell action cancel out. In this situation, the addition of counterterms is not required. These results lead us to conclude that divergent terms contain information about the Legendre 
transformed generating functional, and thus they have to be taken into account.

Note that, unlike the usual Legendre transform prescription Eq.(12), the Legendre transformation Eq. (30) can also be successfully employed to compute the transformed functional in the cases of $\nu$ integer and in particular of $\nu=0$. This follows from the fact that the expansion in powers of the distance to the boundary is done only after having carried out the Legendre transformation.

Another important observation to be made is that the generalized Legendre transform prescription Eq. (30) has no coefficient to be fixed 'by hand'. In this extended formalism, the conjugated field of $A_{0}$ is just $\tilde{A}_{0}$, and its precise form is fixed in a natural way by the generalized Legendre transform prescription.

In general, we will show that when considering the generalized AdS/CFT prescription Eqs.29 31), we remove all the difficulties that arise from the usual prescription Eqs.(12, 28). In particular, we will show that the new constraints for which irregular modes propagate in the bulk are fixed in a natural way by the generalized AdS/CFT prescription Eqs. 29. 31), and thus they need no longer to be fixed 'by hand', as it is the case when considering the usual formalism. Another point that we will show is that such new constraints are the same for which the divergent local terms of the on-shell action cancel out. In this case, we do not need to add any counterterms.

Summarizing, in this work we are considering two formulations which, at first sight, seem to be completely different of each other. One of them is performed in global coordinates, and it constructs the canonical energies and requires that they are conserved, positive and finite. The another one is performed in Poincaré coordinates, and it finds the boundary CFT two-point functions by making use of the generalized AdS/CFT prescription. The fact that, as we will show, these two formulations give rise to exactly the same constraints for which irregular modes propagate in the bulk, can be considered as a remarkable non-trivial result, and a strong evidence in support of our conjectures that the canonical energy is the natural one to be considered in the AdS/CFT correspondence context, and that the generalized AdS/CFT prescription Eqs.(29-31) is needed in order to map to the border all the information contained in the bulk.

In the following sections, we will present our results in detail. In particular, we will construct the canonical energies and perform the quantization of the scalar field theory on AdS. We will also compute the boundary CFT's two-point functions by making use of the generalized AdS/CFT prescription Eqs.(29-31), and show that all the difficulties that arise from the usual AdS/CFT prescription Eqs. 12, 28) can be removed in this new context. And finally, in Section 4, we will show that our results arising from all calculations are in complete agreement, with only one remarkable exception, namely the case in which the conformal dimension of the boundary CFT reaches the unitarity 
bound for the scalar field theory, and becomes independent of the effective mass. This is a very puzzling phenomenon which has no bulk counterpart.

\section{The Canonical Energy}

In this section, we formulate Dirichlet, Neumann and mixed boundary-value problems for a real massive scalar field theory on $A d S_{d+1}$ in both minimally and non-minimally coupled cases. Then, for each one of these boundary-value problems, we compute the Noether current corresponding to time displacements in global coordinates, and show that it is conserved, in the sense that its covariant divergence vanishes. By requiring that the corresponding 'conserved' charge, i.e. the canonical energy, is actually conserved, we will find regular and irregular solutions, and new constraints for which the irregular ones propagate in the bulk. We will also show that for such regular and irregular modes, and only for them, the canonical energy is also positive and finite, thus leading to a consistent quantization of the scalar field theory on AdS. We leave for the next section to show that, contrary to what happens to the constraint Eq.(26), which arises on considering the metrical energy, the new constraints for which irregular modes propagate in the bulk can be reproduced through a generalized AdS/CFT prescription.

We consider a $\mathrm{d}+1$ dimensional space $\mathcal{M}$ with metric $g_{\mu \nu}$ of 'mostly plus' signature. Let $x^{\mu}$ be coordinates on $\mathcal{M}$. We begin by analyzing the non-minimally coupled case, and leave the minimally coupled case to be considered later. The usual matter contribution to the action of a system containing a massive real scalar field non-minimally coupled to the metric $g_{\mu \nu}$ is given by

$$
I_{0}=-\frac{1}{2} \int_{\mathcal{M}} d^{d+1} x \sqrt{g}\left[g^{\mu \nu} \partial_{\mu} \phi \partial_{\nu} \phi+\left(m^{2}+\varrho R\right) \phi^{2}\right],
$$

where $m$ is the mass of the scalar field, $R$ is the Ricci scalar corresponding to $g_{\mu \nu}$ and $\varrho$ is an arbitrary coupling coefficient between the scalar field and the metric.

We consider $\mathcal{M}$ as foliated by a one-parameter family of $\mathrm{d}$ dimensional timelike surfaces $\partial \mathcal{M}_{\rho}$ homeomorphic to the timelike boundary $\partial \mathcal{M}$. Here $\rho$ is a real parameter and in particular we assume that $\partial \mathcal{M}_{\rho} \rightarrow \partial \mathcal{M}$ as $\rho \rightarrow \rho_{0}$. We refer to $\partial \mathcal{M}_{\rho}$ as the boundary to the interior region $\mathcal{M}_{\rho}$. In general, the limit $\rho \rightarrow \rho_{0}$ will be taken only at the end of the calculations.

It can be shown that, in order for the action $I_{0}$ to be stationary under an infinitesimal variation of the metric 


$$
g_{\mu \nu} \rightarrow g_{\mu \nu}+\delta g_{\mu \nu}
$$

the metric and certain of its normal derivatives must fixed at $\partial \mathcal{M}_{\rho}$. In order to have a well-defined variational principle, we must add to $I_{0}$ a surface term of the form [48]

$$
\varrho \int_{\partial \mathcal{M}_{\rho}} d^{d} x \sqrt{h} K \phi^{2}
$$

which accounts for the terms of the variation containing derivatives of the metric. Here $h_{\mu \nu}$ is the induced metric on $\partial \mathcal{M}_{\rho}$, and $K$ is the trace of the extrinsic curvature. It is given by

$$
K=-\nabla_{\mu} n^{\mu},
$$

where $n_{\mu}$ is the outward pointing unit normal vector to $\partial \mathcal{M}_{\rho}$. The surface term Eq.(34) is just the natural extension of the Gibbons-Hawking term [47] which is added to the Einstein-Hilbert action in order to have a well-defined variational principle.

Then, the resulting matter contribution to the action reads

$$
I_{D}=-\frac{1}{2} \int_{\mathcal{M}_{\rho}} d^{d+1} x \sqrt{g}\left[g^{\mu \nu} \partial_{\mu} \phi \partial_{\nu} \phi+\left(m^{2}+\varrho R\right) \phi^{2}\right]+\varrho \int_{\partial \mathcal{M}_{\rho}} d^{d} x \sqrt{h} K \phi^{2} .
$$

The reason for the notation ' $I_{D}$ ' to refer to the matter contribution will be clarified later. It can be shown that, as expected, $I_{D}$ is stationary under the variation Eq.(33) when the metric is fixed at the boundary.

So far, we have concentrated only on infinitesimal variations of the metric, as in Eq.(33). In order to consider boundary-value problems on the scalar field, we have to analyze the transformation properties of the action under infinitesimal variations of the scalar field as follows

$$
\phi \rightarrow \phi+\delta \phi .
$$

Under the variation above, the matter contribution $I_{D}$ transforms as

$$
\delta_{\phi} I_{D}=-\int_{\partial \mathcal{M}_{\rho}} d^{d} x \sqrt{h}\left(\partial_{n} \phi-2 \varrho K \phi\right) \delta \phi,
$$

where $\partial_{n} \phi$ is the Lie derivative of $\phi$ along $n_{\mu}$, and it is given by

$$
\partial_{n} \phi=n^{\mu} \partial_{\mu} \phi .
$$


Note that, in Eq.(38), the absence of a bulk contribution is due to the equation of motion

$$
\nabla^{2} \phi-\left(m^{2}+\varrho R\right) \phi=0
$$

The variation Eq. (38) shows that the action $I_{D}$ is stationary for a Dirichlet boundary condition which fixes the value of the scalar field $\phi$ at $\partial \mathcal{M}_{\rho}$, namely

$$
\left.\delta \phi\right|_{\partial \mathcal{M}_{\rho}}=0 \quad \text { (Dirichlet) }
$$

This is the reason why we called this action ' $I_{D}$ '.

In this way, we have found an action which has a well-defined variational principle when both the metric and the scalar field are fixed at the boundary. However, for more general boundary conditions, new surface terms must be added to the matter contribution $I_{D}$. On doing this, we define the following action

$$
I_{M, I}=I_{D}+\int_{\partial \mathcal{M}_{\rho}} d^{d} x \sqrt{h} \phi \partial_{n} \phi
$$

which differs from $I_{D}$ only by a surface term. It can be shown that the new surface term does not spoil the property of having a well-defined variational principle under Eq. (33) when the metric is fixed at the boundary. Besides, it can be verified that, under the variation of the scalar field Eq.(37), the action $I_{M, I}$ transforms as

$$
\delta_{\phi} I_{M, I}=\int_{\partial \mathcal{M}_{\rho}} d^{d} x \sqrt{h} \phi \delta \psi^{I}
$$

where the absence of a bulk contribution is due to the equation of motion. The field $\psi^{I}$ in the equation above is defined as

$$
\psi^{I}=\partial_{n} \phi+2 \varrho K \phi .
$$

Then, the action $I_{M, I}$ is stationary under a boundary condition which fixes $\psi^{I}$ at $\partial \mathcal{M}_{\rho}$, and we call this as 'Type I' mixed boundary condition. Namely

$$
\left.\delta \psi^{I}\right|_{\partial \mathcal{M}_{\rho}}=0 \quad \text { (Type I mixed) } .
$$

Note that there exists a one-parameter family of Type I mixed boundary conditions.

\footnotetext{
${ }^{6}$ Note that we could also have considered the boundary condition $\left.\left(\partial_{n} \phi-2 \varrho K \phi\right)\right|_{\partial \mathcal{M}_{\rho}}=0$. However, this kind of boundary condition plays no role in the present formalism. Analogous observations hold for the remaining boundary conditions to be considered in this paper.
} 
So far, we have considered the non-minimally coupled case. For the minimally coupled case $\varrho=0$, the Dirichlet boundary condition Eq.(41) stays as a Dirichlet boundary condition. On the other hand, for $\varrho=0$ the Type I mixed boundary condition in Eq.(45) becomes a Neumann boundary condition, as noted from Eq.(44). Thus, at first sight, it seems that for the minimally coupled case only Dirichlet and Neumann boundary conditions are possible. However, this is not the case. For the particular case of $\varrho=0$, consider the following action

$$
I_{M, I I}=\left.I_{D}\right|_{\varrho=0}-\lambda \int_{\partial \mathcal{M}_{\rho}} d^{d} x \sqrt{h}\left(\partial_{n} \phi\right)^{2},
$$

where $\lambda$ is an arbitrary real coefficient. The action $I_{M, I I}$ differs from $\left.I_{D}\right|_{\varrho=0}$ only on a surface term, and to the best of our knowledge it has been considered for the first time in [7], in the context of the AdS/CFT correspondence. As in the cases considered before, it can be shown that the new surface term does not spoil the property of having a well-defined variational principle under Eq.(33) when the metric is fixed at the boundary. Furthermore, under the variation of the scalar field Eq.(37) the action $I_{M, I I}$ transforms as

$$
\delta_{\phi} I_{M, I I}=-\int_{\partial \mathcal{M}_{\rho}} d^{d} x \sqrt{h} \partial_{n} \phi \delta \psi^{I I}
$$

where, as before, the absence of a bulk contribution is due to the equation of motion. The field $\psi^{I I}$ in Eq.(47) is defined as

$$
\psi^{I I}=\phi+2 \lambda \partial_{n} \phi
$$

Thus, the action $I_{M, I I}$ is stationary under a boundary condition which fixes $\psi^{I I}$ at $\partial \mathcal{M}_{\rho}$, and we call this as 'Type II' mixed boundary condition. Namely

$$
\left.\delta \psi^{I I}\right|_{\partial \mathcal{M}_{\rho}}=0 \quad \text { (Type II mixed) }
$$

Note that, as in the case of Type I mixed boundary conditions, there exists a oneparameter family of Type II mixed boundary conditions. In the case of Type I mixed boundary conditions, the corresponding parameter is just the coupling coefficient between the field and the metric $\varrho$, whereas for Type II mixed boundary conditions the corresponding parameter is $\lambda$, which plays for the Type II mixed boundary conditions a role which is analogous to the one played by the coupling coefficient $\varrho$ for Type I mixed boundary conditions. 
In this way, we have computed the actions for scalar field theory on a curved space which correspond to Dirichlet, Neumann and mixed boundary conditions on $\partial \mathcal{M}_{\rho}$ in both the minimally and non-minimally coupled cases, and that have also a well-defined variational principle under Eq.(33) when the metric is fixed at the boundary.

From now on, we concentrate on the specific case of $d+1$ dimensional Anti-de Sitter spaces, whose metric in global coordinates reads

$$
d s^{2}=\frac{1}{\cos ^{2} \rho}\left(-d \tau^{2}+d \rho^{2}+\sin ^{2} \rho d \Omega_{d}^{2}\right) \quad(d \geq 2),
$$

where we have fixed the radius of $A d S_{d+1}$ equal to one. Here, $d \Omega_{d}^{2}$ is the angular element, and $\rho$ and $\tau$ are the radial and time coordinates respectively. They satisfy

$$
\begin{aligned}
0 & \leq \rho<\frac{\pi}{2} \quad(d \geq 2), \\
-\pi & \leq \tau<\pi .
\end{aligned}
$$

In order to avoid closed timelike curves, we follow 41] and pass to the universal covering space CAdS by setting

$$
-\infty<\tau<\infty .
$$

We consider that the boundary-value problems analyzed before are formulated on surfaces of fixed radial coordinate $\rho$. Such surfaces are homeomorphic to the boundary at $\rho \rightarrow \rho_{0}=\frac{\pi}{2}$. The surface forming an outer normal vector is given by

$$
n_{\mu}=\frac{1}{\cos \rho} \delta_{\mu}^{(\rho)}
$$

and then we find

$$
K=-\frac{1}{\sin \rho}\left(d-\cos ^{2} \rho\right) .
$$

The first step to perform the quantization of the scalar field theory on AdS is to compute the Noether currents corresponding to time displacements. In order to do this, we consider a Killing vector $\xi^{\mu}$ of the form

$$
\xi^{\mu}=-\delta_{(\tau)}^{\mu},
$$


where we have included a minus sign because of the "mostly plus" signature of the metric. Then, we perform the isometry

$$
x^{\mu} \longrightarrow x^{\mu}+\sigma \xi^{\mu},
$$

where $\sigma$ is an infinitesimal constant parameter. The variations of the actions $I_{D}, I_{M, I}$ and $I_{M, I I}$ under the isometry above are of the generic form

$$
\delta_{\xi} I=\sigma \int_{\partial \mathcal{M}_{\rho}} d^{d} x \sqrt{h} n_{\mu} J^{\mu}
$$

where $J^{\mu}$ is the Noether current, and since Noether currents are sensitive to the addition of boundary terms to the action, we expect to have different Noether currents corresponding to the actions $I_{D}, I_{M, I}$ and $I_{M, I I}$. Making use of the equation of motion Eq.(40), and simplifying the calculations by noting that $g^{\mu \nu}, R, n_{\mu}$ and $K$ are invariant under Eq.(57),[] we find the following Noether currents corresponding to the time displacement Eq.(57)

$$
\begin{gathered}
J_{D}^{\mu}=-\Theta_{\tau}^{\mu}-\varrho\left[\delta_{\tau}^{\mu} \nabla_{\nu}\left(K n^{\nu} \phi^{2}\right)-K n^{\mu} \partial_{\tau} \phi^{2}\right] \\
J_{M, I}^{\mu}=-\Theta_{\tau}^{\mu}-\varrho\left[\delta_{\tau}^{\mu} \nabla_{\nu}\left(K n^{\nu} \phi^{2}\right)-K n^{\mu} \partial_{\tau} \phi^{2}\right] \\
-\frac{1}{2}\left[\delta_{\tau}^{\mu} \nabla^{2} \phi^{2}-\partial^{\mu} \partial_{\tau} \phi^{2}\right], \\
J_{M, I I}^{\mu}=-\left.\Theta_{\tau}^{\mu}\right|_{\varrho=0}-\lambda\left[n^{\nu} \partial^{\mu} \phi \partial_{\nu} \partial_{\tau} \phi+n^{\nu} \partial_{\nu} \phi \partial^{\mu} \partial_{\tau} \phi\right. \\
\left.-\delta_{\tau}^{\mu} \nabla_{\nu}\left(n^{\alpha} \partial_{\alpha} \phi \partial^{\nu} \phi\right)\right], \\
\Theta_{\mu \nu}=\partial_{\mu} \phi \partial_{\nu} \phi-\frac{1}{2} g_{\mu \nu}\left[g^{\alpha \beta} \partial_{\alpha} \phi \partial_{\beta} \phi+\left(m^{2}+\varrho R\right) \phi^{2}\right] .
\end{gathered}
$$

Making use of the equation of motion, we can show that the above Noether currents are conserved, as expected. Note that the surface terms in the actions $I_{D}, I_{M, I}$ and $I_{M, I I}$ introduce 'improvement' terms to the Noether currents which are analogous to the 'improvement' term that the coupling between the scalar field and the metric introduces to the stress-energy tensor (see Eq.(24)). The canonical energies are obtained from the $J^{\tau}$ component of the Noether currents Eqs.(59-61), and they read

\footnotetext{
${ }^{7}$ Note that $n^{\mu}$ is not a true vector, and its variation includes an extra deviation term.
} 


$$
\begin{aligned}
E_{D} & =-\int d^{d} x \sqrt{g}\left[\Theta^{\tau}{ }_{\tau}+\varrho \nabla_{\mu}\left(K n^{\mu} \phi^{2}\right)\right], \\
E_{M, I} & =-\int d^{d} x \sqrt{g}\left[\Theta^{\tau}{ }_{\tau}+\varrho \nabla_{\mu}\left(K n^{\mu} \phi^{2}\right)+\frac{1}{2}\left(\nabla^{2} \phi^{2}-\partial^{\tau} \partial_{\tau} \phi^{2}\right)\right], \\
E_{M, I I} & =-\int d^{d} x \sqrt{g}\left\{\left.\Theta^{\tau}{ }_{\tau}\right|_{\varrho=0}+\lambda\left[\partial_{\tau}\left(\partial^{\tau} \phi \partial_{n} \phi\right)-\nabla_{\mu}\left(\partial^{\mu} \phi \partial_{n} \phi\right)\right]\right\},
\end{aligned}
$$

where the integration is carried out over the spatial coordinates.

The next step is to solve the equation of motion Eq.(40) in global coordinates. This has been done in [6] 35] 36] [40] [41, and we recall here the main results in order to introduce some notation and make some relevant comments. The solution can be expanded in modes as

$$
\phi=\sum_{\omega l\{m\}}\left[a_{\omega l\{m\}} \phi_{\omega l\{m\}}+a_{\omega l\{m\}}^{*} \phi_{\omega l\{m\}}^{*}\right] \quad(d \geq 3),
$$

where the $a_{\omega l\{m\}}$ 's are complex coefficients and the modes $\phi_{\omega l\{m\}}$ are of the form

$$
\phi_{\omega l\{m\}}=N_{\omega l} e^{-i \omega \tau} Y_{l\{m\}}\left(\Omega_{d}\right) G_{\omega l}(\rho) \quad(d \geq 3) .
$$

Here the $\omega$ 's are real numbers to be determined later, $N_{\omega l}$ are normalization coefficients and the $Y_{l\{m\}}$ 's are spherical harmonics in $\mathbf{S}^{d-1}$. We will analyze here the case $d \geq 3$, but the case $d=2$ is analogous and we will comment on it later in this section.

The radial part of the equation of motion reads

$$
\partial_{\rho}\left[(\tan \rho)^{d-1} \partial_{\rho} G_{\omega l}(\rho)\right]=\left[\frac{M^{2}(\varrho)}{\cos ^{2} \rho}-\omega^{2}+l(l+d-2) \frac{1}{\sin ^{2} \rho}\right](\tan \rho)^{d-1} G_{\omega l}(\rho),
$$

where $M^{2}(\varrho)$ is the effective mass on Eq.(16). It is convenient to write

$$
G_{\omega l}(\rho)=(\sin \rho)^{l}(\cos \rho)^{\Delta} F_{\omega l}(\rho),
$$

where $\Delta$ is an arbitrary real parameter. We choose

$$
\Delta(\Delta-d)=M^{2}(\varrho)
$$

and the solutions of this equation are the coefficients $\Delta_{ \pm}(\varrho)$ in Eq.(18). After straightforward algebra, Eq. (68) can be written as 


$$
0=x(1-x) \frac{d^{2} F_{\omega l}^{ \pm}}{d x^{2}}+\left[c-\left(a_{ \pm}+b_{ \pm}+1\right) x\right] \frac{d F_{\omega l}^{ \pm}}{d x}-a_{ \pm} b_{ \pm} F_{\omega l}^{ \pm}
$$

where

$$
\begin{gathered}
x=\sin ^{2} \rho \\
a_{ \pm}=\frac{1}{2}\left[l+\Delta_{ \pm}(\varrho)-\omega\right], \\
b_{ \pm}=\frac{1}{2}\left[l+\Delta_{ \pm}(\varrho)+\omega\right], \\
c=l+\frac{d}{2} .
\end{gathered}
$$

As pointed out in 35 36], for each choice $\Delta_{+}(\varrho)$ or $\Delta_{-}(\varrho)$ only one of the two independent solutions of Eq.(71) is regular at the origin in $\rho=0$, and it is given by

$$
F_{\omega l}^{ \pm}(\rho)={ }_{2} F_{1}\left(a_{ \pm}, b_{ \pm} ; c ; \sin ^{2} \rho\right)
$$

where ${ }_{2} F_{1}(a, b ; c ; x)$ is the hypergeometric function. The solution of Eq.(68) which is regular at the origin 8 can be expanded in powers of $\cos \rho$ by making use of the identities presented in Appendix A. It is important to perform the analysis by considering the cases of $\nu$ not integer, $\nu$ integer but not zero and $\nu=0$ separately. After some calculations, we arrive at the following series expansions of the regular radial solution $G_{\omega l}(\rho)$.

i) For $\nu \notin \mathbf{Z}$,

$$
\begin{aligned}
G_{\omega l}(\rho) & =(\sin \rho)^{l}(\cos \rho)^{\Delta_{-}(\varrho)} \frac{\Gamma\left(l+\frac{d}{2}\right) \Gamma(\nu) \Gamma(1-\nu)}{\Gamma\left(a_{+}\right) \Gamma\left(b_{+}\right) \Gamma\left(a_{-}\right) \Gamma\left(b_{-}\right)} \\
& \times \sum_{n \geq 0} \frac{1}{n !}(\cos \rho)^{2 n}\left[\frac{\Gamma\left(n+a_{-}\right) \Gamma\left(n+b_{-}\right)}{\Gamma(n+1-\nu)}-\frac{\Gamma\left(n+a_{+}\right) \Gamma\left(n+b_{+}\right)}{\Gamma(n+1+\nu)}(\cos \rho)^{2 \nu}\right] .
\end{aligned}
$$

\footnotetext{
${ }^{8}$ Note that, at first sight, we are in presence of two regular solutions of Eq.(68), namely $(\sin \rho)^{l}(\cos \rho)^{\Delta_{+}(\varrho)} F_{\omega l}^{+}(\rho)$ and $(\sin \rho)^{l}(\cos \rho)^{\Delta_{-}(\varrho)} F_{\omega l}^{-}(\rho)$. However, Eq.(68) is a second order differential equation, and taking into account that we have discarded its non-regular solution, we expect that only one solution is left. In fact, the identity $(\cos \rho)^{\Delta_{+}(\varrho)} F_{\omega l}^{+}(\rho)=(\cos \rho)^{\Delta_{-}(\varrho)} F_{\omega l}^{-}(\rho)$ can be shown by using standard properties of the hypergeometric function. As far as we know, this was not pointed out before.
} 
ii) For $\nu=0$,

$$
\begin{aligned}
G_{\omega l}(\rho) & =-(\sin \rho)^{l}(\cos \rho)^{\Delta_{+}(\varrho)} \frac{\Gamma\left(l+\frac{d}{2}\right)}{\Gamma\left(a_{+}\right) \Gamma\left(b_{+}\right) \Gamma\left(a_{-}\right) \Gamma\left(b_{-}\right)} \\
& \times \sum_{n \geq 0} \frac{1}{(n !)^{2}} \Gamma\left(n+a_{+}\right) \Gamma\left(n+b_{+}\right)\left[2 \ln \cos \rho+u_{n}\left(a_{-}, b_{-}, 0\right)\right](\cos \rho)^{2 n},
\end{aligned}
$$

where $u_{n}(a, b, m)$ is given by Eq.(175).

iii) For $\nu \in \mathbf{Z}, \nu>0$,

$$
\begin{aligned}
& G_{\omega l}(\rho)=(\sin \rho)^{l}(\cos \rho)^{\Delta_{-}(\varrho)} \frac{\Gamma\left(l+\frac{d}{2}\right)}{\Gamma\left(a_{+}\right) \Gamma\left(b_{+}\right) \Gamma\left(a_{-}\right) \Gamma\left(b_{-}\right)} \\
& \times {\left[\sum_{n=0}^{\nu-1} \frac{(-1)^{n}}{n !} \Gamma(\nu-n) \Gamma\left(n+a_{-}\right) \Gamma\left(n+b_{-}\right)(\cos \rho)^{2 n}\right.} \\
&-(-1)^{\nu}(\cos \rho)^{2 \nu} \sum_{n \geq 0} \frac{\Gamma\left(n+a_{+}\right) \Gamma\left(n+b_{+}\right)}{\Gamma(n+1) \Gamma(n+1+\nu)} \\
&\left.\times\left[2 \ln \cos \rho+u_{n}\left(a_{-}, b_{-}, \nu\right)\right](\cos \rho)^{2 n}\right] .
\end{aligned}
$$

The next step is to analyze the conditions for which the canonical energies Eqs. 63 . 65) are conserved, finite and positive. As in the case of the metrical energy, the canonical energy is actually conserved if there is no flux of the corresponding conserved current through the boundary at $\rho \rightarrow \rho_{0}=\frac{\pi}{2}$. This condition gives rise to regular and irregular modes, just as it happens to the usual formalism involving the metrical energy along the lines of [35][36] [40]. Thus, regular and irregular modes arise on considering either the metrical and the canonical energies. The conditions (for $n=0,1,2, \cdots$ )

$$
\begin{array}{lll}
a_{+}=-n, & \text { or } \quad b_{+}=-n & \text { (regular modes) } \\
a_{-}=-n, & \text { or } \quad b_{-}=-n & \text { (irregular modes) }
\end{array}
$$

quantize $\omega$ as follows 


$$
\begin{array}{ll}
\left|\omega_{n}^{+}\right|=l+\Delta_{+}(\varrho)+2 n & \text { (regular modes) } \\
\left|\omega_{n}^{-}\right|=l+\Delta_{-}(\varrho)+2 n & \text { (irregular modes) }
\end{array}
$$

and give rise to regular and irregular solutions, whose asymptotic behavior is of the form

$$
\begin{array}{ll}
G_{n l}^{+} \sim \hat{\epsilon}^{\Delta_{+}(\varrho)} & \text { (regular modes) } \\
G_{n l}^{-} \sim \hat{\epsilon}^{\Delta_{-}(\varrho)} & \text { (irregular modes) }
\end{array}
$$

where $\hat{\epsilon}=\cos \rho$.

The main result of this section will be the derivation of the constraints for which the canonical energies Eqs.63-65) are actually conserved, allowing regular and irregular modes to propagate in the bulk. The conditions for which the flux of the Noether currents corresponding to the canonical energies vanish can be written for a superposition of two modes as:

i) For Dirichlet boundary conditions,

$$
0=\lim _{\hat{\epsilon} \rightarrow 0}\left[\hat{\epsilon}^{-(d-1)} G_{\omega l}\left(\partial_{\hat{\epsilon}} G_{\omega^{\prime} l}-2 \varrho d \hat{\epsilon}^{-1} G_{\omega^{\prime} l}\right)\right] .
$$

ii) For Type I mixed boundary conditions,

$$
0=\lim _{\hat{\epsilon} \rightarrow 0}\left[\hat{\epsilon}^{-(d-1)} G_{\omega l}\left(\partial_{\hat{\epsilon}} G_{\omega^{\prime} l}+2 \varrho d \hat{\epsilon}^{-1} G_{\omega^{\prime} l}\right)\right] .
$$

iii) For Type II mixed boundary conditions,

$$
0=\lim _{\hat{\epsilon} \rightarrow 0}\left[\left.\hat{\epsilon}^{-(d-1)} \partial_{\hat{\epsilon}} G_{\omega l}\left(G_{\omega^{\prime} l}-2 \lambda \hat{\epsilon} \partial_{\hat{\epsilon}} G_{\omega^{\prime} l}\right)\right|_{\varrho=0}\right] .
$$

As in the cases of the scalar product Eq.(4) and the metrical energy which is constructed out of the conserved current Eq.(25), for the canonical energies the regular modes propagate for any real $\nu \geq 0$, whereas the irregular ones propagate when the constraint Eq. (20) is satisfied. There is nothing new here. Note also that the Breitenlohner-Freedman bound Eq.(21) still holds, as expected. The key result is that, instead of the usual constraint Eq.(26) corresponding to irregular modes propagating in 
the bulk when the metrical energy is considered, for the case of the canonical energies there arise new constraints which can be computed from the conditions Eqs. 81.83). Making use of Eqs.(75), we find that the new constraints read as follows:

i) Dirichlet boundary conditions.

In this case we find the constraint

$$
\varrho=\frac{1}{2 d} \Delta_{-}(\varrho)
$$

which has two possible solutions

$$
\varrho_{D}^{ \pm}=\frac{d-1}{8 d}\left[1 \pm \sqrt{1+\left(\frac{4 m}{d-1}\right)^{2}}\right] .
$$

ii) Type I mixed boundary conditions.

The constraint corresponding to this case reads

$$
\varrho=-\frac{1}{2 d} \Delta_{-}(\varrho)
$$

and has solutions

$$
\varrho_{M, I}^{ \pm}=-\frac{3 d+1}{8 d}\left[1 \mp \sqrt{1+\left(\frac{4 m}{3 d+1}\right)^{2}}\right] .
$$

iii) Type II mixed boundary conditions.

In this case, the constraint reads

$$
\lambda=\lambda_{M, I I}
$$

where

$$
\lambda_{M, I I}=\frac{1}{2 \Delta_{-}(0)} .
$$

The key result that we will show in the next section is that, unlike the usual constraint Eq.(26) for which the metrical energy is conserved for irregular modes propagating in 
the bulk, the new constraints Eqs.(84, 86, 88) can be reproduced in a natural way by means of a generalized AdS/CFT prescription, which also contains the information about the constraint Eq.(20). We will also show in the next section that the constraints Eqs.(20, 84, 86, 88) are the ones for which the divergent local terms of the on-shell actions cancel out. In this situation, the addition of counterterms is not required.

As a consequence of Eq.(78), the regular and irregular modes can be written in terms of Jacobi polynomials as follows

$$
G_{n l}^{ \pm}(\rho)=(\sin \rho)^{l}(\cos \rho)^{\Delta_{ \pm}(\varrho)} n ! \frac{\Gamma\left(l+\frac{d}{2}\right)}{\Gamma\left(l+\frac{d}{2}+n\right)} P_{n}^{\left(l+\frac{d}{2}-1, \pm \nu\right)}(\cos 2 \rho) .
$$

For regular and irregular modes, the general solution Eq.(66) reads

$$
\phi^{ \pm}=\sum_{n l\{m\}}\left[a_{n l\{m\}}^{ \pm} \phi_{n l\{m\}}^{ \pm}+a_{n l\{m\}}^{ \pm *} \phi_{n l\{m\}}^{ \pm *}\right] \quad(d \geq 3)
$$

where

$$
\phi_{n l\{m\}}^{ \pm}=N_{n l}^{ \pm} e^{-i \omega_{n}^{ \pm} \tau} Y_{l\{m\}}\left(\Omega_{d}\right) G_{n l}^{ \pm}(\rho) \quad(d \geq 3) .
$$

The normalization coefficients $N_{n l}^{ \pm}$can be computed from the orthogonality properties of the Jacobi polynomials and the spherical harmonics, and their precise values are not relevant for our present purposes.

Finally, we will show that for regular and irregular modes and for the constraints Eqs.(20, 84, 86, 88), the canonical energies Eqs.(63-65) are positive and finite, thus leading to a consistent quantization of the scalar field theory on AdS. We define the field $\phi^{\prime \pm}$ through

$$
\phi^{ \pm}=(\cos \rho)^{\Delta_{ \pm}(\varrho)} \phi^{\prime \pm} .
$$

This means that $\phi^{\prime+}\left(\phi^{\prime-}\right)$ approaches the boundary as a constant for regular (irregular) solutions.

Using Eqs.63-65, 70, 93) and integrating by parts, we find that

$$
\begin{aligned}
E_{D}^{ \pm}=E_{V}^{ \pm}+\int d \Omega_{d} & (\cos \rho)^{2\left(\Delta_{ \pm}(\varrho)-1\right)}(\tan \rho)^{d-1} \\
& \times\left.\cos \rho \sin \rho\left(-\frac{\Delta_{ \pm}(\varrho)}{2}+\frac{\varrho d}{\sin ^{2} \rho}-\frac{\varrho}{\tan ^{2} \rho}\right)\left(\phi^{\prime \pm}\right)^{2}\right|_{\rho=\frac{\pi}{2}},
\end{aligned}
$$




$$
\begin{gathered}
E_{M, I}^{ \pm}=E_{V}^{ \pm}+\int d \Omega_{d}(\cos \rho)^{2\left(\Delta_{ \pm}(\varrho)-1\right)}(\tan \rho)^{d-1} \\
\times\left[\cos \rho \sin \rho\left(\frac{\Delta_{ \pm}(\varrho)}{2}+\frac{\varrho d}{\sin ^{2} \rho}-\frac{\varrho}{\tan ^{2} \rho}\right)\left(\phi^{\prime \pm}\right)^{2}\right. \\
\left.\quad-\cos ^{2} \rho \phi^{\prime \pm} \partial_{\rho} \phi^{\prime \pm}\right]\left.\right|_{\rho=\frac{\pi}{2}}, \\
E_{M, I I}^{ \pm}=\left.E_{V}^{ \pm}\right|_{\varrho=0}+\int d \Omega_{d}(\cos \rho)^{2\left(\Delta_{ \pm}(0)-1\right)}(\tan \rho)^{d-1} \\
\times\left[\lambda \cos ^{3} \rho\left(\partial_{\rho} \phi^{\prime \pm}\right)^{2}-2 \lambda \Delta_{ \pm}(0) \cos ^{2} \rho \sin \rho \phi^{\prime \pm} \partial_{\rho} \phi^{\prime \pm}\right. \\
\left.+\frac{\Delta_{ \pm}(0)}{2} \cos \rho \sin \rho\left(-1+2 \lambda \Delta_{ \pm}(0) \sin \rho\right)\left(\phi^{\prime \pm}\right)^{2}\right]\left.\right|_{\rho=\frac{\pi}{2}}
\end{gathered}
$$

where the volume term is the same as the one for the metrical energy as computed in [35] [36] 40], and it is given by

$$
\begin{aligned}
E_{V}^{ \pm} & =\frac{1}{2} \int d \Omega_{d} \int_{0}^{\frac{\pi}{2}} d \rho(\cos \rho)^{2\left(\Delta_{ \pm}(\varrho)-1\right)}(\tan \rho)^{d-1} \\
& \times\left[\cos ^{2} \rho\left(\left(\partial_{\tau} \phi^{\prime \pm}\right)^{2}+\left(\partial_{\rho} \phi^{\prime \pm}\right)^{2}+\Delta_{ \pm}(\varrho)\left(\phi^{\prime \pm}\right)^{2}\right)\right. \\
& \left.+\frac{1}{\tan ^{2} \rho}\left(\left(\partial_{\theta_{1}} \phi^{\prime \pm}\right)^{2}+\sum_{i=2}^{d-2} \frac{1}{\prod_{j=1}^{i-1} \sin ^{2} \theta_{j}}\left(\partial_{\theta_{i}} \phi^{\prime \pm}\right)^{2}+\frac{1}{\prod_{j=1}^{d-2} \sin ^{2} \theta_{j}}\left(\partial_{\varphi} \phi^{\prime \pm}\right)^{2}\right)\right]
\end{aligned}
$$

where $\varphi$ and $\theta_{i}(1 \leq i \leq d-2)$ are spherical coordinates with $0 \leq \varphi<2 \pi$ and $0 \leq \theta_{i}<\pi$. Note that the term $\sum_{i=2}^{d-2} \frac{1}{\prod_{j=1}^{i-1} \sin ^{2} \theta_{j}}\left(\partial_{\theta_{i}} \phi^{\prime \pm}\right)^{2}$ in the equation above is not present for $d=3$. We stress the fact that the difference between two canonical energies, or between a canonical energy and the metrical one, is just a surface term, as expected. From Eqs.(94 97), it is straightforward to compute the conditions for which the canonical energies are positive and finite, and the procedure is analogous to the one employed in [35] [36] 40] in the context of the formulation involving the metrical energy. The volume term Eq.(97) is manifestly positive. Note that for the solution $\Delta_{+}(\varrho)$ it is also convergent for any value of $\nu$, whereas for the solution $\Delta_{-}(\varrho)$ it is convergent only when $\nu$ satisfies the constraint Eq.(20). On the other hand, the surface terms 
in Eqs.94-96) vanish for the solution $\Delta_{+}(\varrho)$, but they are divergent for the solution $\Delta_{-}(\varrho)$, unless we set the constraints Eqs. (84, 86, 88) which cancel the divergent terms out. Thus, the constraints which make the canonical energies to be conserved are the same for which they are also positive and finite.

Once the Cauchy problem has been solved, we can perform the quantization in the usual way by regarding the coefficients $a_{n l\{m\}}^{ \pm}$and $a_{n l\{m\}}^{ \pm *}$ in Eq.(91) as operators satisfying the commuting relations

$$
\begin{gathered}
{\left[a_{n l\{m\}}^{ \pm}, a_{n^{\prime} l^{\prime}\left\{m^{\prime}\right\}}^{ \pm}\right]=\left[a_{n l\{m\}}^{ \pm *}, a_{n^{\prime} l^{\prime}\left\{m^{\prime}\right\}}^{ \pm *}\right]=0} \\
{\left[a_{n l\{m\}}^{ \pm}, a_{n^{\prime} l^{\prime}\left\{m^{\prime}\right\}}^{ \pm *}\right]=\delta_{n n^{\prime}} \delta_{l l^{\prime}} \delta_{\{m\}\left\{m^{\prime}\right\}} .}
\end{gathered}
$$

In particular, for the cases Eqs.(85, 87, 89) and the masses Eq.(20), which allow for irregular modes to propagate in the bulk as well as the regular ones, there exist two possible consistent quantizations of the scalar field theory on AdS. From the AdS/CFT correspondence point of view, this means that for such cases we must have two different boundary CFT's. This topic will be considered in detail in the next section.

So far, we have analyzed the case $d \geq 3$. We close this section by discussing the case $d=2$. The most important difference between the case $d=2$ and the higher dimensional ones is that for $d=2$ the angular part of the equation of motion Eq.(40) has a normalized solution of the form $\frac{1}{\sqrt{2 \pi}} e^{-i l \varphi}$ (for $0 \leq \varphi<2 \pi$ and $l \in \mathbf{Z}$ ) instead of the spherical harmonics $Y_{l\{m\}}\left(\Omega_{d}\right)$ (see Eq.(67)). Note that, unlike the higher dimensional cases, for $d=2$ the angular number $l$ can also be negative. But for negative $l$ the radial solution Eq.(69) is divergent at the origin. Thus, for $d=2$ we have to make use of $|l|$ instead of $l$. The remaining calculations are analogous to the ones performed in the higher dimensional cases.

So far, we have developed a consistent quantization of the scalar field theory on the AdS bulk. We have claimed in Section 2 that the usual AdS/CFT prescription has no information about the constraints for which irregular modes propagate in the bulk in the usual quantization along the lines of 355 36 40]. In the next section, we will consider a generalized AdS/CFT prescription which, as we will show, gives rise in a natural way to the new constraints Eqs.(20, 84, 86, 88) for which irregular modes propagate in the bulk when the canonical energy is considered instead of the metrical one. In this way, we will be able to remove all the difficulties that we have discussed in Section 2 regarding the usual formulation. 


\section{The Generalized AdS/CFT Prescription}

In this section, we present the generalized version of the usual AdS/CFT prescription. It means that, instead of Eqs.(12, 14, 28), we will make use of Eqs.(29-31). The main goal will be to show that this generalized prescription gives rise, in a natural way, to the constraints Eqs.(20, 84, 86, 88) for which the irregular modes propagate in the AdS bulk when the canonical energy is considered instead of the metrical one. This provides strong evidence in support of our conjectures that the canonical energy is the natural one to be considered in the context of the AdS/CFT correspondence, and that the generalized prescription Eqs.(29-31) is needed in order to map to the border all the information contained in the bulk. We will show that the constraints Eqs. 20, 84, 86, 88) are the ones for which the Legendre transformation Eq.(30) interpolates between the conformal dimensions $\Delta_{+}(\varrho)$ and $\Delta_{-}(\varrho)$, and for which the divergent local terms in the action cancel out. In this situation, there is no need to add any counterterms. An interesting result that we will also find is that there exist particular cases for which the unitarity bound is reached and the conformal dimension becomes independent of the mass. This phenomenon has no bulk counterpart.

We work throughout this section in the Euclidean representation of the $A d S_{d+1}$ in Poincaré coordinates described by the half space $x_{0}>0, x_{i} \in \mathbf{R}$ with metric

$$
d s^{2}=\frac{1}{x_{0}^{2}} \sum_{\mu=0}^{d} d x^{\mu} d x^{\mu},
$$

where, as in the previous section, we have fixed the radius of $A d S_{d+1}$ equal to one. We consider the space as foliated by a family of surfaces $x_{0}=\epsilon$ whose corresponding outward pointing unit normal vector is

$$
n_{\mu}=\left(-\epsilon^{-1}, \mathbf{0}\right)
$$

from which we find the following trace of the extrinsic curvature

$$
K=-d .
$$

We want to formulate boundary-value problems for the scalar field on the surfaces $x_{0}=\epsilon$, and then, according to the prescription in [4], perform the limit $\epsilon \rightarrow 0$ only at the end of calculations. Performing a Wick rotation, we write the actions Eqs.(36, 42, 46) as 


$$
\begin{aligned}
I_{D} & =\frac{1}{2} \int d^{d+1} x \sqrt{g}\left[g^{\mu \nu} \partial_{\mu} \phi \partial_{\nu} \phi+\left(m^{2}+\varrho R\right) \phi^{2}\right]-\varrho \int d^{d} x \sqrt{h} K_{\epsilon} \phi_{\epsilon}^{2}, \\
I_{M, I} & =I_{D}-\int d^{d} x \sqrt{h} \phi_{\epsilon} \partial_{n} \phi_{\epsilon}, \\
I_{M, I I} & =\left.I_{D}\right|_{\varrho=0}+\lambda \int d^{d} x \sqrt{h}\left(\partial_{n} \phi_{\epsilon}\right)^{2},
\end{aligned}
$$

where $\phi_{\epsilon}$ is the value of the field at $x_{0}=\epsilon$, and $\partial_{n} \phi_{\epsilon}$ is its Lie derivative along $n_{\mu}$ given by $\partial_{n} \phi=n^{\mu} \partial_{\mu} \phi$. Using the equation of motion Eq.(40), it can easily be shown that the above actions are stationary under the infinitesimal variation $\phi \rightarrow \phi+\delta \phi$ for Dirichlet, Type I mixed and Type II mixed boundary conditions on the scalar field at the surface $x_{0}=\epsilon$, respectively. Namely

$$
\begin{aligned}
I_{D} & \rightarrow \delta \phi_{\epsilon}=0 & & \text { (Dirichlet) }, \\
I_{M, I} & \rightarrow \delta \psi_{\epsilon}^{I}=0 & & \text { (Type I mixed) }, \\
I_{M, I I} & \rightarrow \delta \psi_{\epsilon}^{I I}=0 & & \text { (Type II mixed) },
\end{aligned}
$$

where the fields $\psi^{I}$ and $\psi^{I I}$ are defined as in Eqs.(44, 48).

Integrating by parts and making use of the equation of motion, the actions Eqs. (103. 105) can be written as the following pure-surface terms

$$
\begin{aligned}
I_{D} & =\frac{1}{2} \int d^{d} x \sqrt{h} \phi_{\epsilon}\left(\partial_{n} \phi_{\epsilon}-2 \varrho K_{\epsilon} \phi_{\epsilon}\right), \\
I_{M, I} & =-\frac{1}{2} \int d^{d} x \sqrt{h} \phi_{\epsilon} \psi_{\epsilon}^{I}, \\
I_{M, I I} & =\frac{1}{2} \int d^{d} x \sqrt{h} \partial_{n} \phi_{\epsilon} \psi_{\epsilon}^{I I} .
\end{aligned}
$$

Note that in the action $I_{D}$, the field $\partial_{n} \phi_{\epsilon}-2 \varrho K_{\epsilon} \phi_{\epsilon}$ must be written in terms of the boundary data $\phi_{\epsilon}$. In the case of $I_{M, I}, \phi_{\epsilon}$ is to be written in terms of the data $\psi_{\epsilon}^{I}$. And in the action $I_{M, I I}$, we have to write $\partial_{n} \phi_{\epsilon}$ in terms of $\psi_{\epsilon}^{I I}$. In all calculations, we make use of the solution of the equation of motion which is regular at $x_{0} \rightarrow \infty$, namely [4]

$$
\phi(x)=\int \frac{d^{d} k}{(2 \pi)^{d}} e^{-i \vec{k} \cdot \vec{x}} x_{0}^{\frac{d}{2}} b(\vec{k}) K_{\nu}\left(k x_{0}\right),
$$


where $\vec{x}=\left(x^{1}, \ldots, x^{d}\right), k=|\vec{k}|, K_{\nu}$ is the modified Bessel function, and $\nu$ is given by Eq.(19). From the equation above, we also get

$$
\partial_{n} \phi(x)=-\int \frac{d^{d} k}{(2 \pi)^{d}} e^{-i \vec{k} \cdot \vec{x}} x_{0}^{\frac{d}{2}} b(\vec{k})\left[\left(\frac{d}{2}+\nu\right) K_{\nu}\left(k x_{0}\right)-k x_{0} K_{\nu+1}\left(k x_{0}\right)\right] .
$$

In the following sub-sections, we will consider each boundary condition separately.

\subsection{Dirichlet Boundary Condition}

Here we will consider the generalized AdS/CFT prescription Eqs.(29) 31) in the particular case of Dirichlet boundary conditions, and the key result will be to reproduce, in a natural way, the constraints Eqs. (20, 84) obtained in the bulk.

Let $\phi_{\epsilon}(\vec{k})$ be the Fourier transform of the Dirichlet boundary value of the field $\phi_{\epsilon}(\vec{x})$. From Eq.(112) we find

$$
b(\vec{k})=\frac{\epsilon^{-\frac{d}{2}} \phi_{\epsilon}(\vec{k})}{K_{\nu}(k \epsilon)},
$$

and, inserting this into Eq.(113), we write $\partial_{n} \phi_{\epsilon}$ in terms of the boundary data $\phi_{\epsilon}$ as

$$
\partial_{n} \phi_{\epsilon}(\vec{x})=-\int d^{d} y \phi_{\epsilon}(\vec{y}) \int \frac{d^{d} k}{(2 \pi)^{d}} e^{-i \vec{k} \cdot(\vec{x}-\vec{y})}\left[\frac{d}{2}+\nu-k \epsilon \frac{K_{\nu+1}(k \epsilon)}{K_{\nu}(k \epsilon)}\right] .
$$

Using this together with Eq.(102), we can write the action Eq.(109) as

$$
\begin{aligned}
I_{D}\left[\phi_{\epsilon}\right]=-\frac{1}{2} & \int d^{d} x d^{d} y \sqrt{h} \phi_{\epsilon}(\vec{x}) \phi_{\epsilon}(\vec{y}) \\
& \times \int \frac{d^{d} k}{(2 \pi)^{d}} e^{-i \vec{k} \cdot(\vec{x}-\vec{y})}\left[\frac{d}{2}+\nu-2 \varrho d-k \epsilon \frac{K_{\nu+1}(k \epsilon)}{K_{\nu}(k \epsilon)}\right] .
\end{aligned}
$$

This is only one of the two functionals which contain the information about the boundary CFT's. The another one is obtained by performing the Legendre transformation Eq. (30), with the identifications $A_{\epsilon}(\vec{x}) \equiv \phi_{\epsilon}(\vec{x}), \tilde{A}_{\epsilon}(\vec{x}) \equiv \tilde{\phi}_{\epsilon}(\vec{x})$, on the action above. This is written as

$$
\mathcal{J}_{D}\left[\phi_{\epsilon}, \tilde{\phi}_{\epsilon}\right]=I_{D}\left[\phi_{\epsilon}\right]-\int d^{d} x \sqrt{h} \phi_{\epsilon}(\vec{x}) \tilde{\phi}_{\epsilon}(\vec{x})
$$


which, after setting $\frac{\partial \mathcal{J}_{D}}{\partial \phi_{\epsilon}}=0$, gives rise to the identity ?

$$
\phi_{\epsilon}=-\frac{1}{\frac{d}{2}+\nu-2 \varrho d-k \epsilon \frac{K_{\nu+1}(k \epsilon)}{K_{\nu}(k \epsilon)}} \tilde{\phi}_{\epsilon} .
$$

Then, the Legendre transform of $I_{D}\left[\phi_{\epsilon}\right]$ reads

$$
\tilde{I}_{D}\left[\tilde{\phi}_{\epsilon}\right]=\frac{1}{2} \int d^{d} x d^{d} y \sqrt{h} \tilde{\phi}_{\epsilon}(\vec{x}) \tilde{\phi}_{\epsilon}(\vec{y}) \int \frac{d^{d} k}{(2 \pi)^{d}} e^{-i \vec{k} \cdot(\vec{x}-\vec{y})} \frac{1}{\frac{d}{2}+\nu-2 \varrho d-k \epsilon \frac{K_{\nu+1}(k \epsilon)}{K_{\nu}(k \epsilon)}}
$$

Both functionals $I_{D}$ and $\tilde{I}_{D}$ are needed in order to map to the boundary all the information contained in the bulk. Each one of the fields $\phi_{\epsilon}$ and $\tilde{\phi}_{\epsilon}$ will couple, after performing the limit $\epsilon \rightarrow 0$ in a proper way and through the prescriptions Eqs.(29, 31), to the corresponding boundary conformal operator. The transformed functional $\tilde{I}_{D}\left[\tilde{\phi}_{\epsilon}\right]$ is to be contrasted with the usual one Eq.(13), which contains only a non-local term. In the transformed functional $\tilde{I}_{D}\left[\tilde{\phi}_{\epsilon}\right]$ we will find, after performing a series expansion in powers of $\epsilon$, both local and non-local terms, just as it happens to the original functional Eq.(116). An important result will be that the conformal dimension $\Delta_{-}(\varrho)$ arises precisely when the divergent local terms cancel out, showing that they contain information about the leading non-local term, and need to be taken into account. And the key result that we will show is that such cancellation of divergent terms arises, precisely, for the constraints Eqs. 20, 84) obtained in the bulk. Note that, unlike what happens to the usual transformed functional Eq.(13), the functional $\tilde{I}_{D}\left[\tilde{\phi}_{\epsilon}\right]$ contains no coefficient to be fixed 'by hand'. Besides, the particular case of $\nu=0$, which as explained in Section 2 gives rise to some difficulties in the usual formulation, will be successfully analyzed in this generalized context, through the simple procedure of performing a series expansion of the ratio $k \epsilon \frac{K_{1}(k \epsilon)}{K_{0}(k \epsilon)}$ in powers of $\epsilon$.

We begin by concentrating on the case of $\nu$ not integer. Note that, by making use of the series expansion

$$
\begin{aligned}
k \epsilon \frac{K_{\nu+1}(k \epsilon)}{K_{\nu}(k \epsilon)}=2 \nu[ & \left(1-\frac{1}{4 \nu(1-\nu)}(k \epsilon)^{2}+O\left(\epsilon^{4}\right)\right) \\
& \left.+\left(2^{-2 \nu} \frac{\Gamma(1-\nu)}{\Gamma(1+\nu)}(k \epsilon)^{2 \nu}+O\left(\epsilon^{2 \nu+2}\right)\right)\right],
\end{aligned}
$$

\footnotetext{
${ }^{9}$ Note that the global minus sign at the r.h.s of Eq.(118) is just conventional and has no physical meaning, because it cancels out on computing the transformed functional.
} 
we can write Eq.(118) as

$$
\phi_{\epsilon}=-\left[\Delta_{-}(\varrho)-2 \varrho d+\frac{1}{2(1-\nu)}(k \epsilon)^{2}-2^{1-2 \nu} \frac{\Gamma(1-\nu)}{\Gamma(\nu)}(k \epsilon)^{2 \nu}+\cdots\right]^{-1} \tilde{\phi}_{\epsilon}
$$

where the dots stand for higher order terms in $\epsilon$. The functionals $I_{D}$ and $\tilde{I}_{D}$ read

$$
\begin{aligned}
I_{D}\left[\phi_{\epsilon}\right]= & -\frac{1}{2} \int d^{d} x d^{d} y \phi_{\epsilon}(\vec{x}) \phi_{\epsilon}(\vec{y}) \epsilon^{-d} \\
& \times \int \frac{d^{d} k}{(2 \pi)^{d}} e^{-i \vec{k} \cdot(\vec{x}-\vec{y})}\left[\Delta_{-}(\varrho)-2 \varrho d+\frac{1}{2(1-\nu)}(k \epsilon)^{2}\right. \\
& \left.-2^{1-2 \nu} \frac{\Gamma(1-\nu)}{\Gamma(\nu)}(k \epsilon)^{2 \nu}+\cdots\right] \\
\tilde{I}_{D}\left[\tilde{\phi}_{\epsilon}\right]=\frac{1}{2} \int d^{d} x d^{d} y \tilde{\phi}_{\epsilon}(\vec{x}) \tilde{\phi}_{\epsilon}(\vec{y}) \epsilon^{-d} & \quad \times \int \frac{d^{d} k}{(2 \pi)^{d}} e^{-i \vec{k} \cdot(\vec{x}-\vec{y})}\left[\Delta_{-}(\varrho)-2 \varrho d+\frac{1}{2(1-\nu)}(k \epsilon)^{2}\right. \\
& \left.\quad-2^{1-2 \nu} \frac{\Gamma(1-\nu)}{\Gamma(\nu)}(k \epsilon)^{2 \nu}+\cdots\right]^{-1} .
\end{aligned}
$$

We consider first the situation in which the constraint Eq.(84) is not satisfied, i.e. we have $\varrho \neq \varrho_{D}^{ \pm}$, with $\varrho_{D}^{ \pm}$given by Eq.(85). Then Eq.(121) reads

$$
\phi_{\epsilon}=-\left[\frac{1}{\Delta_{-}(\varrho)-2 \varrho d}+\cdots\right] \tilde{\phi}_{\epsilon} \quad\left(\varrho \neq \varrho_{D}^{ \pm}\right)
$$

and the fields $\phi_{\epsilon}$ and $\tilde{\phi}_{\epsilon}$ have the same asymptotic behavior. This means that, in this particular case, the Legendre transformation interpolates between boundary operators of the same conformal dimension (which will happen to be $\Delta_{+}(\varrho)$, as expected). This is a novel situation, since the usual Legendre transform prescription Eq.(12) only interpolates between different conformal dimensions. However, our result is in agreement with the fact that, when the constraint Eq. (84) is not satisfied, only regular modes propagate in the bulk, thus leading to expect to find only one conformal dimension at the boundary (namely $\Delta_{+}(\varrho)$ ). In this situation, the divergent local terms in Eqs.(122, 
123) do not cancel out. They encode the information, through Eq.(124), that in this case the Legendre transformation interpolates between boundary operators of the same conformal dimension. Integrating over $\vec{k}$ in Eqs.(122, 123) we get

$$
\begin{aligned}
I_{D}^{\varrho \neq \varrho_{D}^{ \pm}}\left[\phi_{\epsilon}\right]= & \text { divergent local terms } \\
& -\frac{\nu(\varrho)}{\pi^{\frac{d}{2}}} \frac{\Gamma\left(\Delta_{+}(\varrho)\right)}{\Gamma(\nu(\varrho))} \int d^{d} x d^{d} y \phi_{\epsilon}(\vec{x}) \phi_{\epsilon}(\vec{y}) \frac{\epsilon^{-2 \Delta_{-}(\varrho)}}{|\vec{x}-\vec{y}|^{2 \Delta_{+}(\varrho)}}+\cdots, \\
\tilde{I}_{D}^{\varrho \neq \varrho_{D}^{ \pm}}\left[\tilde{\phi}_{\epsilon}\right]= & \text { divergent local terms } \\
& -\frac{\nu(\varrho)}{\pi^{\frac{d}{2}}} \frac{1}{\left[\Delta_{-}(\varrho)-2 \varrho d\right]^{2}} \frac{\Gamma\left(\Delta_{+}(\varrho)\right)}{\Gamma(\nu(\varrho))} \int d^{d} x d^{d} y \tilde{\phi}_{\epsilon}(\vec{x}) \tilde{\phi}_{\epsilon}(\vec{y}) \frac{\epsilon^{-2 \Delta_{-}(\varrho)}}{|\vec{x}-\vec{y}|^{2 \Delta_{+}(\varrho)}} \\
& +\cdots,
\end{aligned}
$$

where the dots stand for higher order terms in $\epsilon$. The non-local term in Eq.(125) is the same as the one analyzed in [⿴囗十 $[5]$, with an effective mass Eq.(16). In this case, the generalized AdS/CFT prescription Eq.(29) reduces to the usual Dirichlet prescription Eq.(28). The non-local term in Eq.(126) differs from the one in Eq.(125) only by a normalization factor. Taking the limits

$$
\begin{aligned}
& \lim _{\epsilon \rightarrow 0} \epsilon^{-\Delta_{-}(\varrho)} \phi_{\epsilon}(\vec{x})=\phi_{0}(\vec{x}), \\
& \lim _{\epsilon \rightarrow 0} \epsilon^{-\Delta_{-}(\varrho)} \tilde{\phi}_{\epsilon}(\vec{x})=\tilde{\phi}_{0}(\vec{x}),
\end{aligned}
$$

to go to the border, and making use of the AdS/CFT prescription

$$
\begin{aligned}
\exp \left(-I_{D}\left[\phi_{0}\right]\right) & \equiv\left\langle\exp \left(\int d^{d} x \mathcal{O}_{D}(\vec{x}) \phi_{0}(\vec{x})\right)\right\rangle, \\
\exp \left(-\tilde{I}_{D}\left[\tilde{\phi}_{0}\right]\right) & \equiv\left\langle\exp \left(\int d^{d} x \tilde{\mathcal{O}}_{D}(\vec{x}) \tilde{\phi}_{0}(\vec{x})\right)\right\rangle,
\end{aligned}
$$

we find the following boundary two-point functions

$$
\begin{aligned}
\left\langle\mathcal{O}_{D}^{\varrho \neq \varrho_{D}^{ \pm}}(\vec{x}) \mathcal{O}_{D}^{\varrho \neq \varrho_{D}^{ \pm}}(\vec{y})\right\rangle & =\frac{2 \nu(\varrho)}{\pi^{\frac{d}{2}}} \frac{\Gamma\left(\Delta_{+}(\varrho)\right)}{\Gamma(\nu(\varrho))} \frac{1}{|\vec{x}-\vec{y}|^{2 \Delta_{+}(\varrho)}} \\
& + \text { divergences }
\end{aligned}
$$




$$
\begin{aligned}
\left\langle\tilde{\mathcal{O}}_{D}^{\varrho \neq \varrho_{D}^{ \pm}}(\vec{x}) \tilde{\mathcal{O}}_{D}^{\varrho \neq \varrho_{D}^{ \pm}}(\vec{y})\right\rangle & =\frac{2 \nu(\varrho)}{\pi^{\frac{d}{2}}} \frac{1}{\left[\Delta_{-}(\varrho)-2 \varrho d\right]^{2}} \frac{\Gamma\left(\Delta_{+}(\varrho)\right)}{\Gamma(\nu(\varrho))} \frac{1}{|\vec{x}-\vec{y}|^{2 \Delta_{+}(\varrho)}} \\
& + \text { divergences }
\end{aligned}
$$

where the divergences are due to the fact that the functionals Eqs.(125, 126) contain divergent local terms. In this situation, both conformal operators $\mathcal{O}_{D}^{\varrho \neq \varrho_{D}^{ \pm}}$and $\tilde{\mathcal{O}}_{D}^{\varrho \neq \varrho_{D}^{ \pm}}$ have the same conformal dimension $\Delta_{+}(\varrho)$, as expected from the analysis in Section 3 which establishes that for $\varrho \neq \varrho_{D}^{ \pm}$only regular modes propagate in the bulk when the canonical energy is taken into account. From Eqs.(127, 128), we conclude that near the border the classical modes, which encode the information about the boundary CFT, behave as $x_{0}^{\Delta_{-}(\varrho)}$, also as expected from the results in Section 3 . The Legendre transformation interpolates between conformal operators of the same conformal dimension. We remark that this information is encoded in the fact that the divergent local terms of the functionals $I_{D}^{\varrho \neq \varrho_{D}^{ \pm}}\left[\phi_{\epsilon}\right]$ and $\tilde{I}_{D}^{\varrho \neq \varrho_{D}^{ \pm}}\left[\tilde{\phi}_{\epsilon}\right]$ do not cancel out. In this case, the only difference between the boundary two-point functions Eqs.(131, 132) is just a normalization factor.

Now we consider the case in which the constraint Eq.(84) is satisfied, i.e. we have $\varrho=\varrho_{D}^{ \pm}$. In this situation we expect, from the analysis in Section 3 , to find two different boundary CFT's, namely the ones of conformal dimensions $\Delta_{+}(\varrho)$ and $\Delta_{-}(\varrho)$. Unlike the case of $\varrho \neq \varrho_{D}^{ \pm}$, in the situation $\varrho=\varrho_{D}^{ \pm}$we must analyze two different cases, namely the ones in which the constraint Eq.(20) is, or not, also satisfied. We begin by concentrating on the situation in which the constraint Eq.(20) is also satisfied (with the particular case $\nu=0$ naturally excluded, since we are considering here that $\nu$ is not an integer).

Let us first analyze the original functional $I_{D}\left[\phi_{\epsilon}\right]$. Integrating over $\vec{k}$ in Eq. 122) we find

$$
I_{D}^{\varrho=\varrho_{D}^{ \pm} ; \nu<1}\left[\phi_{\epsilon}\right]=-\frac{\nu(\varrho)}{\pi^{\frac{d}{2}}} \frac{\Gamma\left(\Delta_{+}(\varrho)\right)}{\Gamma(\nu(\varrho))} \int d^{d} x d^{d} y \phi_{\epsilon}(\vec{x}) \phi_{\epsilon}(\vec{y}) \frac{\epsilon^{-2 \Delta_{-}(\varrho)}}{|\vec{x}-\vec{y}|^{2 \Delta_{+}(\varrho)}}+\cdots,
$$

where the dots stand for higher order terms in $\epsilon$. Notice that the only, and crucial difference between Eqs. (125, 133), is that now the divergent local terms have cancelled out. This fact will acquire great relevance when we consider the transformed functional $\tilde{I}_{D}\left[\tilde{\phi}_{\epsilon}\right]$. 
Taking the limit Eq.(127), we find the two-point function

$$
\left\langle\mathcal{O}_{D}^{\varrho=\varrho_{D}^{ \pm} ; \nu<1}(\vec{x}) \mathcal{O}_{D}^{\varrho=\varrho_{D}^{ \pm} ; \nu<1}(\vec{y})\right\rangle=\frac{2 \nu(\varrho)}{\pi^{\frac{d}{2}}} \frac{\Gamma\left(\Delta_{+}(\varrho)\right)}{\Gamma(\nu(\varrho))} \frac{1}{|\vec{x}-\vec{y}|^{2 \Delta_{+}(\varrho)}}
$$

which contains the same non-local term as the two-point function Eq.(131), as expected from the fact that the functionals Eqs.(125, 133) share the same leading non-local term, but that, unlike Eq.(131), does not contain any divergences. Thus, when the constraint Eq.(84) is satisfied, the divergences disappear. The conformal operator $\mathcal{O}_{D}^{\varrho=\varrho_{D}^{ \pm} ; \nu<1}$ has conformal dimension $\Delta_{+}(\varrho)$, and the classical bulk modes to which it couples behave close to the boundary as $x_{0}^{\Delta_{-}(\varrho)}$.

So far, we have been able to reproduce only the conformal dimension $\Delta_{+}(\varrho)$. From the analysis in Section 3, we expect to find also the conformal dimension $\Delta_{-}(\varrho)$. Let us show that it arises on considering the transformed functional $\tilde{I}_{D}\left[\tilde{\phi}_{\epsilon}\right]$. Making use of Eqs.(20, 84), we note that Eq.(121) can be written as

$$
\phi_{\epsilon}=\left[2^{2 \nu-1} \frac{\Gamma(\nu)}{\Gamma(1-\nu)}(k \epsilon)^{-2 \nu}+\cdots\right] \tilde{\phi}_{\epsilon},
$$

where the dots stand for higher order terms in $\epsilon$. In this case, the fields $\phi_{\epsilon}$ and $\tilde{\phi}_{\epsilon}$ have different asymptotic behaviors, and thus the Legendre transformation Eq.(117) interpolates between two different conformal dimensions. Since, as we have shown, $\phi_{\epsilon}$ corresponds to the conformal dimension $\Delta_{+}(\varrho)$, it is clear from Eq. (135) that $\tilde{\phi}_{\epsilon}$ corresponds to the missing conformal dimension $\Delta_{-}(\varrho)$, as expected. The information that the Legendre transformation interpolates between two different conformal dimensions is encoded in the fact that, in this case, the divergent local terms in Eqs.(122, 123) cancel out. The constraints Eqs. 20, 84) arise, in this generalized AdS/CFT prescription, in a natural way, as anticipated. Integrating over $\vec{k}$ in Eq.(123) we get

$$
\begin{aligned}
\tilde{I}_{D}^{\varrho=\varrho_{D}^{ \pm} ; \nu<1}\left[\tilde{\phi}_{\epsilon}\right]=- & \frac{1}{4 \pi^{\frac{d}{2}}} \frac{\Gamma\left(\Delta_{-}(\varrho)\right)}{\Gamma(1-\nu(\varrho))} \int d^{d} x d^{d} y \tilde{\phi}_{\epsilon}(\vec{x}) \tilde{\phi}_{\epsilon}(\vec{y}) \frac{\epsilon^{-2 \Delta_{+}(\varrho)}}{|\vec{x}-\vec{y}|^{2 \Delta_{-}(\varrho)}} \\
& +\cdots,
\end{aligned}
$$

where the dots stand for higher order terms in $\epsilon$. Notice that the fact that the divergent local terms have cancelled out has given rise to the presence, in the transformed functional $\tilde{I}_{D}\left[\tilde{\phi}_{\epsilon}\right]$, of a new (with respect to the one in Eq.(126)) leading non-local term, 
which, as we will show, corresponds to a boundary CFT of conformal dimension $\Delta_{-}(\varrho)$, as expected.

Taking the limit

$$
\lim _{\epsilon \rightarrow 0} \epsilon^{-\Delta_{+}(\varrho)} \tilde{\phi}_{\epsilon}(\vec{x})=\tilde{\phi}_{0}(\vec{x}),
$$

we find the following two-point function

$$
\left\langle\tilde{\mathcal{O}}_{D}^{\varrho=\varrho_{D}^{ \pm} ; \nu<1}(\vec{x}) \tilde{\mathcal{O}}_{D}^{\varrho=\varrho_{D}^{ \pm} ; \nu<1}(\vec{y})\right\rangle=\frac{1}{2 \pi^{\frac{d}{2}}} \frac{\Gamma\left(\Delta_{-}(\varrho)\right)}{\Gamma(1-\nu(\varrho))} \frac{1}{|\vec{x}-\vec{y}|^{2 \Delta_{-}(\varrho)}}
$$

and the conformal operator $\tilde{\mathcal{O}}_{D}^{\varrho=\varrho_{D}^{ \pm} ; \nu<1}$ has conformal dimension $\Delta_{-}(\varrho)$, as expected. Besides, in this situation we note from Eq.(137) that the classical modes which encode the information about the boundary CFT behave asymptotically as $x_{0}^{\Delta_{+}(\varrho)}$. In this way, we have been able, through the generalized AdS/CFT prescription Eqs.229.31, to reproduce in a natural way the constraints Eqs.(20, 84) for which irregular modes propagate in the bulk for Dirichlet boundary conditions and when the canonical energy, instead of the metrical one, is taken into account. As anticipated, we have also shown that irregular modes arise when the divergent local terms of the action cancel out, and that such divergent terms encode the information about the leading non-local terms, in the sense that when they cancel out, and only then, the Legendre transformation Eq.(30) interpolates between different conformal dimensions. In this situation, the addition of counterterms to the on-shell action is not required. Note that our prescription has left no coefficient to be fixed 'by hand'.

We still need to analyze the case in which the constraint Eq.(84) is satisfied, but the constraint Eq. (20) is not, i.e. we have $\varrho=\varrho_{D}^{ \pm}$and $\nu>1$. This is a very particular situation for which, as we will show, the unitarity bound $(d-2) / 2$ arises. We begin by considering the original functional $I_{D}\left[\phi_{\epsilon}\right]$. Integration over $\vec{k}$ in Eq.(122) yields

$$
\begin{aligned}
I_{D}^{\varrho=\varrho_{D}^{ \pm} ; \nu>1}\left[\phi_{\epsilon}\right]= & \text { divergent local terms } \\
& -\frac{\nu(\varrho)}{\pi^{\frac{d}{2}}} \frac{\Gamma\left(\Delta_{+}(\varrho)\right)}{\Gamma(\nu(\varrho))} \int d^{d} x d^{d} y \phi_{\epsilon}(\vec{x}) \phi_{\epsilon}(\vec{y}) \frac{\epsilon^{-2 \Delta_{-}(\varrho)}}{|\vec{x}-\vec{y}|^{2 \Delta_{+}(\varrho)}}+\cdots,
\end{aligned}
$$

and then, unlike the case $\nu<1$ (see Eq.(133)), in this situation the original functional contains divergent local terms, because the leading order in Eq.(122) turns out to be $O\left(\epsilon^{-d+2}\right)$. Taking the limit Eq.(127), we find the two-point function 


$$
\begin{aligned}
& \left\langle\mathcal{O}_{D}^{\varrho=\varrho_{D}^{ \pm} ; \nu>1}(\vec{x}) \mathcal{O}_{D}^{\varrho=\varrho_{D}^{ \pm} ; \nu>1}(\vec{y})\right\rangle=\frac{2 \nu(\varrho)}{\pi^{\frac{d}{2}}} \frac{\Gamma\left(\Delta_{+}(\varrho)\right)}{\Gamma(\nu(\varrho))} \frac{1}{|\vec{x}-\vec{y}|^{2 \Delta_{+}(\varrho)}} \\
& + \text { divergences , }
\end{aligned}
$$

which, unlike the two-point function corresponding to the case $\nu<1$ (see Eq.(134)), also contains divergences. The conformal operator $\mathcal{O}_{D}^{\varrho=\varrho_{D}^{ \pm} ; \nu>1}$ has conformal dimension $\Delta_{+}(\varrho)$, and the classical bulk modes to which it couples behave close to the boundary as $x_{0}^{\Delta_{-}(\varrho)}$.

Now we consider the transformed functional $\tilde{I}_{D}\left[\tilde{\phi}_{\epsilon}\right]$. Integrating over $\vec{k}$ in Eq.(123), we get for $d \geq 3$

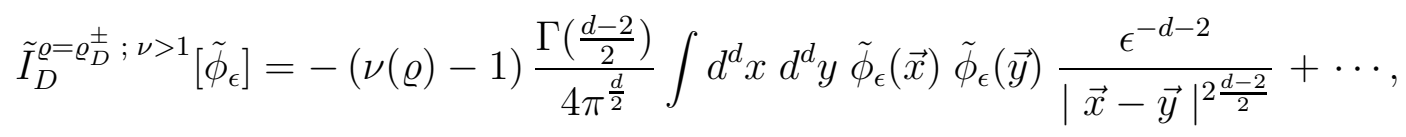

which, unlike the original functional Eq.(139), does not contain any divergent terms. Taking the limit

$$
\lim _{\epsilon \rightarrow 0} \epsilon^{-\frac{d+2}{2}} \tilde{\phi}_{\epsilon}(\vec{x})=\tilde{\phi}_{0}(\vec{x})
$$

we find the boundary two-point function

$$
\left\langle\tilde{\mathcal{O}}_{D}^{\varrho=\varrho_{D}^{ \pm} ; \nu>1}(\vec{x}) \tilde{\mathcal{O}}_{D}^{\varrho=\varrho_{D}^{ \pm} ; \nu>1}(\vec{y})\right\rangle=(\nu(\varrho)-1) \frac{\Gamma\left(\frac{d-2}{2}\right)}{2 \pi^{\frac{d}{2}}} \frac{1}{|\vec{x}-\vec{y}|^{2 \frac{d-2}{2}}},
$$

which does not contain any divergences. The operator $\tilde{\mathcal{O}}_{D}^{\varrho=\varrho_{D}^{ \pm} ; \nu>1}$ has conformal dimension $\frac{d-2}{2}$, which is precisely the unitarity bound. This is a very particular case which does not have any corresponding bulk counterpart. Note from Eq.(142) that the classical backgrounds which couple to $\tilde{\mathcal{O}}_{D}^{\varrho=\varrho_{D}^{ \pm} ; \nu>1}$ behave close to the boundary as $x_{0}^{\frac{d+2}{2}}$, and, as noted from Eq.(75), such modes do not exist in the bulk. From the bulk point of view, the irregular modes propagate for $0 \leq \nu<1$. For $\nu \rightarrow 1$, when the unitarity bound is reached, they just stop propagating. Then, for $\nu>1$, only regular modes propagate in the bulk. On the other hand, from the boundary point of view, the 
conformal dimension $\Delta_{-}(\varrho)$ is present for $0 \leq \nu<1$. For $\nu \rightarrow 1$, when the unitarity bound is reached, it does not disappear. What we find is that the unitarity bound gets saturated, and then, for $\nu>1$, the conformal dimension $\Delta_{-}(\varrho)$ becomes independent of the effective mass Eq.(16). It just stays as $(d-2) / 2$, no matter how much the effective mass grows up. This is a puzzling phenomenon, which has no bulk counterpart, and deserves further studies. We stress the fact that the conformal dimension $(d-2) / 2$ arises only for $d \geq 3$.

So far, we have considered the case of $\nu$ not integer, and now we analyze the case of $\nu$ integer. In such situation, the series expansion of the modified Bessel function contains extra logarithmic terms which must be considered. For the case of $\nu$ integer but non-zero, it is possible to show, following a procedure analogous to the one in [7], that, after integration over $\vec{k}$, the non-local terms result identical to the ones of the case of $\nu$ not integer, thus giving rise to the same boundary two-point functions. On the other hand, the logarithmic divergent local terms, which are not present for $\nu$ not integer, are related to conformal anomalies [22] 24] 25] 29] 30. We will not develop this topic further.

We finish the analysis of the Dirichlet boundary condition by considering in detail the case $\nu=0$. We will show that, in this situation, we reproduce the expected results, namely that the Legendre transformation interpolates between boundary operators of the same conformal dimension $\frac{d}{2}$. As expected, in addition to the usual divergent local terms, we will find logarithmic divergences. We will show that, when the constraint Eq.(84) is satisfied, the usual divergent local terms cancel out, and only the logarithmic divergences survive. Another interesting result that we will show regards the asymptotic behavior of the classical modes which couple to the boundary operator. In general, the case of $\nu=0$ involves a novel situation, in which such classical modes present a logarithmic behavior close to the boundary [1]. A new result that we will show is that, when the constraint Eq.(84) is satisfied, the Legendre transformation interpolates between conformal operators of the same conformal dimension $\frac{d}{2}$, but corresponding to classical bulk modes which differ in their asymptotic behaviors. One of such behaviors is logarithmic, as expected. However, the another one is not, thus resembling the bulk modes which arise for $\nu$ not integer.

Making use of standard identities for the modified Bessel functions, we find

$$
k \epsilon \frac{K_{1}(k \epsilon)}{K_{0}(k \epsilon)}=-\frac{1}{\ln \epsilon}\left[1+\frac{\ln 2-\gamma}{\ln \epsilon}-\frac{\ln k}{\ln \epsilon}+O\left(\epsilon^{2} \ln \epsilon\right)\right],
$$

where $\gamma$ is the Euler constant. Then, the functionals Eqs.(116, 119) can be written as 


$$
\begin{aligned}
& I_{D}^{\nu=0}\left[\phi_{\epsilon}\right]=-\frac{1}{2} \int d^{d} x d^{d} y \phi_{\epsilon}(\vec{x}) \phi_{\epsilon}(\vec{y}) \epsilon^{-d} \\
& \times \int \frac{d^{d} k}{(2 \pi)^{d}} e^{-i \vec{k} \cdot(\vec{x}-\vec{y})}\left[\frac{d}{2}-2 \varrho d+\frac{1}{\ln \epsilon}+\frac{\ln 2-\gamma}{\ln ^{2} \epsilon}-\frac{\ln k}{\ln ^{2} \epsilon}\right. \\
&+\cdots], \\
& \quad \times \int \frac{d^{d} k}{(2 \pi)^{d}} e^{-i \vec{k} \cdot(\vec{x}-\vec{y})}\left[\frac{d}{2}-2 \varrho d+\frac{1}{\ln \epsilon}+\frac{\ln 2-\gamma}{\ln ^{2} \epsilon}-\frac{\ln ^{\nu} k}{\ln ^{2} \epsilon}\right. \\
&\left.+\tilde{\phi}_{\epsilon}\right]=\frac{1}{2} \int d^{d} x d^{d} y \tilde{\phi}_{\epsilon}(\vec{x}) \tilde{\phi}_{\epsilon}(\vec{y}) \epsilon^{-d}
\end{aligned}
$$

where the dots stand for higher order terms in $\epsilon$. Let us first consider the original functional $I_{D}^{\nu=0}\left[\phi_{\epsilon}\right]$. After integration over $\vec{k}$, the leading non-local term reads

$$
I_{D}^{\text {non-local }}\left[\phi_{\epsilon}\right]=-\frac{\Gamma\left(\frac{d}{2}\right)}{4 \pi^{\frac{d}{2}}} \int d^{d} x d^{d} y \phi_{\epsilon}(\vec{x}) \phi_{\epsilon}(\vec{y}) \frac{\epsilon^{-d}}{l n^{2} \epsilon} \frac{1}{|\vec{x}-\vec{y}|^{d}} .
$$

Note that the whole functional $I_{D}^{\nu=0}\left[\phi_{\epsilon}\right]$ contains, in addition, the usual divergent local terms, plus new logarithmic divergences, which are not present for $\nu$ not integer. However, when the constraint Eq.(84) is satisfied, the usual divergent local terms cancel out, and only the logarithmic divergences survive. As pointed out before, they correspond to conformal anomalies, and are to be cancelled out through the addition of proper counterterms.

Taking the limit [7]

$$
\lim _{\epsilon \rightarrow 0}\left(\epsilon^{\frac{d}{2}} \ln \epsilon\right)^{-1} \phi_{\epsilon}(\vec{x})=\phi_{0}(\vec{x}),
$$

in Eq.(147), we find the following boundary two-point function

$$
\left\langle\mathcal{O}_{D}^{\nu=0}(\vec{x}) \mathcal{O}_{D}^{\nu=0}(\vec{y})\right\rangle=\frac{\Gamma\left(\frac{d}{2}\right)}{2 \pi^{\frac{d}{2}}} \frac{1}{|\vec{x}-\vec{y}|^{d}}
$$


Then, the operator $\mathcal{O}_{D}^{\nu=0}$ has conformal dimension $\frac{d}{2}$, as expected. The classical bulk modes approach the boundary as $x_{0}^{\frac{d}{2}} \ln x_{0}$, and we find the logarithmic behavior that we have mentioned before.

Now, we consider the transformed functional $\tilde{I}_{D}^{\nu=0}\left[\tilde{\phi}_{\epsilon}\right]$. Let us first suppose that the constraint Eq.(84) is not satisfied. Then, after integration over $\vec{k}$, the leading non-local term in Eq. (146) reads

$$
\tilde{I}_{D}^{\text {non-local }}\left[\tilde{\phi}_{\epsilon}\right]=-\frac{1}{\left(\frac{d}{2}-2 \varrho d\right)^{2}} \frac{\Gamma\left(\frac{d}{2}\right)}{4 \pi^{\frac{d}{2}}} \int d^{d} x d^{d} y \tilde{\phi}_{\epsilon}(\vec{x}) \tilde{\phi}_{\epsilon}(\vec{y}) \frac{\epsilon^{-d}}{\ln \epsilon} \frac{1}{|\vec{x}-\vec{y}|^{d}}
$$

In addition, the whole functional $\tilde{I}_{D}^{\varrho \neq \varrho_{D}^{ \pm}} ; \nu=0\left[\tilde{\phi}_{\epsilon}\right]$ contains the usual divergent local terms, plus logarithmic divergences.

Taking the limit

$$
\lim _{\epsilon \rightarrow 0}\left(\epsilon^{\frac{d}{2}} \ln \epsilon\right)^{-1} \tilde{\phi}_{\epsilon}(\vec{x})=\tilde{\phi}_{0}(\vec{x}),
$$

we find the boundary two-point function

$$
\left\langle\tilde{\mathcal{O}}_{D}^{\varrho \neq \varrho_{D}^{ \pm} ; \nu=0}(\vec{x}) \tilde{\mathcal{O}}_{D}^{\varrho \neq \varrho_{D}^{ \pm} ; \nu=0}(\vec{y})\right\rangle=\frac{1}{\left(\frac{d}{2}-2 \varrho d\right)^{2}} \frac{\Gamma\left(\frac{d}{2}\right)}{2 \pi^{\frac{d}{2}}} \frac{1}{|\vec{x}-\vec{y}|^{d}} .
$$

Then, the operator $\tilde{\mathcal{O}}_{D}^{\varrho \neq \varrho_{D}^{ \pm}} ; \nu=0$ has conformal dimension $\frac{d}{2}$, and the classical bulk modes approach the boundary as $x_{0}^{\frac{d}{2}} \ln x_{0}$.

Finally, we consider the case in which the constraint Eq.(84) is satisfied. In this situation we find that, after integration over $\vec{k}$, the leading non-local term in Eq. (146) reads

$$
\tilde{I}_{D}^{\text {non-local }}\left[\tilde{\phi}_{\epsilon}\right]=-\frac{\Gamma\left(\frac{d}{2}\right)}{4 \pi^{\frac{d}{2}}} \int d^{d} x d^{d} y \tilde{\phi}_{\epsilon}(\vec{x}) \tilde{\phi}_{\epsilon}(\vec{y}) \epsilon^{-d} \frac{1}{|\vec{x}-\vec{y}|^{d}} .
$$

In addition, the whole functional $\tilde{I}_{D}^{\varrho=\varrho_{D}^{ \pm} ; \nu=0}\left[\tilde{\phi}_{\epsilon}\right]$ contains logarithmic divergences. Note, also, that unlike the previous cases, the above leading non-local term does not contain any logarithmic term in $\epsilon$. Then, instead of Eq.(151), the limit to be taken is

$$
\lim _{\epsilon \rightarrow 0} \epsilon^{-\frac{d}{2}} \tilde{\phi}_{\epsilon}(\vec{x})=\tilde{\phi}_{0}(\vec{x}) \text {. }
$$


Thus, we find the following boundary two-point function

$$
\left\langle\tilde{\mathcal{O}}_{D}^{\varrho=\varrho_{D}^{ \pm} ; \nu=0}(\vec{x}) \tilde{\mathcal{O}}_{D}^{\varrho=\varrho_{D}^{ \pm} ; \nu=0}(\vec{y})\right\rangle=\frac{\Gamma\left(\frac{d}{2}\right)}{2 \pi^{\frac{d}{2}}} \frac{1}{|\vec{x}-\vec{y}|^{d}}
$$

Then, the operator $\tilde{\mathcal{O}}_{D}^{\varrho=\varrho_{D}^{ \pm} ; \nu=0}$ has conformal dimension $\frac{d}{2}$. The classical bulk modes approach the boundary as $x_{0}^{\frac{d}{2}}$, and this is a novel situation, because we have not found the usual logarithmic behavior. As anticipated, when the constraint Eq. (84) is satisfied, the Legendre transformation interpolates between conformal operators of the same conformal dimension $\frac{d}{2}$, but corresponding to classical bulk modes which differ in their asymptotic behaviors.

\subsection{Type I Mixed Boundary Condition}

Now we consider the case of Type I mixed boundary conditions. The analysis is analogous to the one performed in the Dirichlet situation, and in this case we expect, on considering the conformal dimension $\Delta_{-}(\varrho)$, to reproduce the constraint Eq.(86), instead of the one in Eq. (84) corresponding to the Dirichlet boundary condition. Besides, we also expect to find again the constraint Eq.(20), because, according to the results in Section 3, it is common to all boundary conditions.

Let $\psi_{\epsilon}^{I}(\vec{k})$ be the Fourier transform of $\psi_{\epsilon}^{I}(\vec{x})$. Then, from Eqs.(44, 102, 112, 113) we find

$$
b(\vec{k})=-\frac{\epsilon^{-\frac{d}{2}}}{K_{\nu}(k \epsilon)} \frac{\psi_{\epsilon}^{I}(\vec{k})}{\frac{d}{2}+\nu+2 \varrho d-k \epsilon \frac{K_{\nu+1}(k \epsilon)}{K_{\nu}(k \epsilon)}},
$$

and, inserting this into Eq.(112), we write $\phi_{\epsilon}$ in terms of the boundary data $\psi_{\epsilon}^{I}$ as

$$
\phi_{\epsilon}(\vec{x})=-\int d^{d} y \psi_{\epsilon}^{I}(\vec{y}) \int \frac{d^{d} k}{(2 \pi)^{d}} e^{-i \vec{k} \cdot(\vec{x}-\vec{y})} \frac{1}{\frac{d}{2}+\nu+2 \varrho d-k \epsilon \frac{K_{\nu+1}(k \epsilon)}{K_{\nu}(k \epsilon)}} .
$$

Then, the action Eq. 110 can be written as

$$
I_{M, I}\left[\psi_{\epsilon}^{I}\right]=\frac{1}{2} \int d^{d} x d^{d} y \sqrt{h} \psi_{\epsilon}^{I}(\vec{x}) \psi_{\epsilon}^{I}(\vec{y}) \int \frac{d^{d} k}{(2 \pi)^{d}} e^{-i \vec{k} \cdot(\vec{x}-\vec{y})} \frac{1}{\frac{d}{2}+\nu+2 \varrho d-k \epsilon \frac{K_{\nu+1}(k \epsilon)}{K_{\nu}(k \epsilon)}}
$$


We also need to compute the corresponding Legendre transformed functional. This is done by considering Eq.(30) with the identifications $A_{\epsilon}(\vec{x}) \equiv \psi_{\epsilon}^{I}(\vec{x}), \tilde{A}_{\epsilon}(\vec{x}) \equiv \tilde{\psi}_{\epsilon}^{I}(\vec{x})$. Then, the Legendre transformation is of the form

$$
\mathcal{J}_{M, I}\left[\psi_{\epsilon}^{I}, \tilde{\psi}_{\epsilon}^{I}\right]=I_{M, I}\left[\psi_{\epsilon}^{I}\right]-\int d^{d} x \sqrt{h} \psi_{\epsilon}^{I}(\vec{x}) \tilde{\psi}_{\epsilon}^{I}(\vec{x}),
$$

which, after setting $\frac{\partial \mathcal{J}_{M, I}}{\partial \psi_{\epsilon}^{I}}=0$, yields

$$
\psi_{\epsilon}^{I}=\left[\frac{d}{2}+\nu+2 \varrho d-k \epsilon \frac{K_{\nu+1}(k \epsilon)}{K_{\nu}(k \epsilon)}\right] \tilde{\psi}_{\epsilon}^{I} .
$$

Then, the Legendre transform of $I_{M, I}\left[\psi_{\epsilon}^{I}\right]$ is of the form

$$
\begin{aligned}
\tilde{I}_{M, I}\left[\tilde{\psi}_{\epsilon}^{I}\right]=-\frac{1}{2} \int d^{d} x d^{d} y \sqrt{h} \tilde{\psi}_{\epsilon}^{I}(\vec{x}) \tilde{\psi}_{\epsilon}^{I}(\vec{y}) \\
\quad \times \int \frac{d^{d} k}{(2 \pi)^{d}} e^{-i \vec{k} \cdot(\vec{x}-\vec{y})}\left[\frac{d}{2}+\nu+2 \varrho d-k \epsilon \frac{K_{\nu+1}(k \epsilon)}{K_{\nu}(k \epsilon)}\right] .
\end{aligned}
$$

The functionals Eqs. (158, 161) contain the information about the boundary CFT's corresponding to the Type I mixed boundary-value problem. Note that, in this case, the generalized AdS/CFT prescription Eqs.(29, 31) reads

$$
\exp \left(-I_{M, I}\left[\psi_{0}^{I}\right]\right) \equiv\left\langle\exp \left(\int d^{d} x \mathcal{O}_{M, I}(\vec{x}) \psi_{0}^{I}(\vec{x})\right)\right\rangle,
$$

for the original functional, and

$$
\exp \left(-\tilde{I}_{M, I}\left[\tilde{\psi}_{0}^{I}\right]\right) \equiv\left\langle\exp \left(\int d^{d} x \tilde{\mathcal{O}}_{M, I}(\vec{x}) \tilde{\psi}_{0}^{I}(\vec{x})\right)\right\rangle,
$$

for the Legendre transformed one. The calculations are analogous to the ones performed in the Dirichlet case. As expected, the conformal dimension $\Delta_{-}(\varrho)$ arises precisely when the constraints Eqs.(20, 86) are satisfied, and in such situation the divergent local terms cancel out. In this case, the addition of counterterms is not required. An interesting feature of this boundary condition is that the conformal dimension $\Delta_{-}(\varrho)$ arises from the original functional Eq.(158), rather than from its corresponding Legendre transformed functional Eq.(161), which gives rise to the conformal dimension $\Delta_{+}(\varrho)$. This is a novel situation, since, as seen before, in the Dirichlet case it is the transformed functional which gives rise to the conformal dimension $\Delta_{-}(\varrho)$. As before, 
there exist particular situations for which the unitarity bound $(d-2) / 2$ arises. In such cases, the conformal dimension does not depend on the effective mass Eq.(16). Since the calculations are analogous to those considered in the Dirichlet case, we just present the main results in Appendix B.

\subsection{Type II Mixed Boundary Condition}

The analysis of the Type II mixed situation is analogous to the ones performed in the Dirichlet and Type I mixed cases. However, it must be taken into account that, in this case, we have to set $\varrho=0$ in all calculations, because, unlike the cases considered before, the Type II mixed boundary conditions are considered only in the minimally coupled case, as seen in Section 3. Note that now we expect to find the constraint Eq. (88), instead of the ones Eqs. (84, 86) that we have considered so far. Besides, we expect to reproduce the constraint Eq.(20) in the particular case of $\varrho=0$.

Let $\psi_{\epsilon}^{I I}(\vec{k})$ be the Fourier transform of $\psi_{\epsilon}^{I I}(\vec{x})$. From Eqs. (48, 112, 113) we find

$$
b(\vec{k})=\left.\frac{\epsilon^{-\frac{d}{2}}}{K_{\nu}(k \epsilon)} \frac{\psi_{\epsilon}^{I I}(\vec{k})}{1-2 \lambda\left(\frac{d}{2}+\nu\right)+2 \lambda k \epsilon \frac{K_{\nu+1}(k \epsilon)}{K_{\nu}(k \epsilon)}}\right|_{\varrho=0},
$$

and using Eq.(113), the above equation allows to write $\partial_{n} \phi_{\epsilon}$ in terms of the boundary data $\psi_{\epsilon}^{I I}$ as

$$
\partial_{n} \phi_{\epsilon}(\vec{x})=-\left.\int d^{d} y \psi_{\epsilon}^{I I}(\vec{y}) \int \frac{d^{d} k}{(2 \pi)^{d}} e^{-i \vec{k} \cdot(\vec{x}-\vec{y})} \frac{\frac{d}{2}+\nu-k \epsilon \frac{K_{\nu+1}(k \epsilon)}{K_{\nu}(k \epsilon)}}{1-2 \lambda\left(\frac{d}{2}+\nu\right)+2 \lambda k \epsilon \frac{K_{\nu+1}(k \epsilon)}{K_{\nu}(k \epsilon)}}\right|_{\varrho=0} .
$$

Thus, the action Eq.(111) reads

$$
\begin{array}{rl}
I_{M, I I}\left[\psi_{\epsilon}^{I I}\right]=-\frac{1}{2} \int d^{d} & x d^{d} y \sqrt{h} \psi_{\epsilon}^{I I}(\vec{x}) \psi_{\epsilon}^{I I}(\vec{y}) \\
& \times\left.\int \frac{d^{d} k}{(2 \pi)^{d}} e^{-i \vec{k} \cdot(\vec{x}-\vec{y})} \frac{\frac{d}{2}+\nu-k \epsilon \frac{K_{\nu+1}(k \epsilon)}{K_{\nu}(k \epsilon)}}{1-2 \lambda\left(\frac{d}{2}+\nu\right)+2 \lambda k \epsilon \frac{K_{\nu+1}(k \epsilon)}{K_{\nu}(k \epsilon)}}\right|_{\varrho=0} .
\end{array}
$$

Note that, in this case, the Legendre transformation Eq.(30) must be performed with the identifications $A_{\epsilon}(\vec{x}) \equiv \psi_{\epsilon}^{I I}(\vec{x}), \tilde{A}_{\epsilon}(\vec{x}) \equiv \tilde{\psi}_{\epsilon}^{I I}(\vec{x})$. Then, it reads 


$$
\mathcal{J}_{M, I I}\left[\psi_{\epsilon}^{I I}, \tilde{\psi}_{\epsilon}^{I I}\right]=I_{M, I I}\left[\psi_{\epsilon}^{I I}\right]-\int d^{d} x \sqrt{h} \psi_{\epsilon}^{I I}(\vec{x}) \tilde{\psi}_{\epsilon}^{I I}(\vec{x})
$$

which, after setting $\frac{\partial \mathcal{J}_{M, I I}}{\partial \psi_{\epsilon}^{I I}}=0$, gives rise to

$$
\psi_{\epsilon}^{I I}=-\left.\frac{1-2 \lambda\left(\frac{d}{2}+\nu\right)+2 \lambda k \epsilon \frac{K_{\nu+1}(k \epsilon)}{K_{\nu}(k \epsilon)}}{\frac{d}{2}+\nu-k \epsilon \frac{K_{\nu+1}(k \epsilon)}{K_{\nu}(k \epsilon)}} \tilde{\psi}_{\epsilon}^{I I}\right|_{\varrho=0} .
$$

Then, we find the following Legendre transform of $I_{M, I I}\left[\psi_{\epsilon}^{I I}\right]$

$$
\begin{aligned}
\tilde{I}_{M, I I}\left[\tilde{\psi}_{\epsilon}^{I I}\right]=\frac{1}{2} \int d^{d} x d^{d} y \sqrt{h} \tilde{\psi}_{\epsilon}^{I I}(\vec{x}) \tilde{\psi}_{\epsilon}^{I I}(\vec{y}) \\
\quad \times\left.\int \frac{d^{d} k}{(2 \pi)^{d}} e^{-i \vec{k} \cdot(\vec{x}-\vec{y})} \frac{1-2 \lambda\left(\frac{d}{2}+\nu\right)+2 \lambda k \epsilon \frac{K_{\nu+1}(k \epsilon)}{K_{\nu}(k \epsilon)}}{\frac{d}{2}+\nu-k \epsilon \frac{K_{\nu+1}(k \epsilon)}{K_{\nu}(k \epsilon)}}\right|_{\varrho=0} .
\end{aligned}
$$

In this case, the generalized AdS/CFT prescription Eqs.(29, 31) reads

$$
\exp \left(-I_{M, I I}\left[\psi_{0}^{I I}\right]\right) \equiv\left\langle\exp \left(\int d^{d} x \mathcal{O}_{M, I I}(\vec{x}) \psi_{0}^{I I}(\vec{x})\right)\right\rangle
$$

for the original functional, and

$$
\exp \left(-\tilde{I}_{M, I I}\left[\tilde{\psi}_{0}^{I I}\right]\right) \equiv\left\langle\exp \left(\int d^{d} x \tilde{\mathcal{O}}_{M, I I}(\vec{x}) \tilde{\psi}_{0}^{I I}(\vec{x})\right)\right\rangle
$$

for the Legendre transformed one. The calculations are analogous to the ones performed in the previous cases, and, as expected, the conformal dimension $\Delta_{-}(0)$ arises precisely when the constraints Eqs.(20, 88) hold (we recall that the constraint Eq.(20) is to be considered in the particular case of $\varrho=0$ ). Also as expected, when the constraints Eqs.(20, 88) are satisfied we find that the divergent local terms cancel out. As in the former cases, there exist particular situations for which the unitarity bound $(d-2) / 2$ arises. Since the calculations are analogous to those considered in the previous cases, we just present the main results in Appendix B. 


\section{Conclusions}

In this work, we have revisited the formulation of the scalar field theory on AdS spaces and in the AdS/CFT correspondence, in both minimal and non-minimal coupled cases. We have shown that, when quantizing the scalar field on AdS along the lines of 35 36] 40], there arise some constraints which cannot be reproduced through the usual AdS/CFT formalism. In addition, we have found some difficulties regarding the usual prescription for the Legendre transform, namely that it leaves a coefficient to be fixed 'by hand', and that it does not work for $\nu=0$. In order to remove this obstacles, we have considered a rather different formulation both in the bulk and in the AdS/CFT correspondence context.

Regarding the formulation in the bulk, we have argued that the usual energy which is constructed out of the stress-energy tensor, namely the metrical energy, is not the natural one to be considered in the context of the AdS/CFT correspondence. Then, we have proposed a new definition of the energy, which makes use of the Noether current corresponding to time displacements in global coordinates. We have shown that this new energy, namely the canonical energy, depends on the boundary conditions, and we have computed it for Dirichlet, Neumann and mixed boundary-value problems, in both minimally and non-minimally coupled cases. By requiring this energy to be conserved, positive and finite, we have found that the Breitenlohner-Freedman bound still holds, that regular and irregular modes propagate in the bulk as it happens when the quantization makes use of the metrical energy, and that there arise new constraints for which the irregular modes propagate.

Then, we have shown that this new formalism gives rise, in a natural way, to a generalized AdS/CFT prescription in which the source which couples to the boundary conformal operator depends on the selected boundary condition, thus involving a situation which is much more general than the usual one, where only Dirichlet boundary conditions are considered. We have also analyzed a generalized prescription for the Legendre transform that, in fact, makes use of the standard form of the Legendre transformation. This means, in particular, that we transform the whole on-shell action, rather than only the leading non-local term, as in [23]. In this generalized prescription, there is no coefficient to be fixed 'by hand', and the case $\nu=0$ can be analyzed in a natural way.

We have shown that this generalized AdS/CFT prescription gives rise, in a natu-

ral way, to the constraints for which irregular modes propagate in the bulk when the canonical energy is taken into account, thus providing strong evidence in support of our formalism. Another interesting result that we have shown is that, for such constraints, 
the divergent local terms of the on-shell action cancel out, and the Legendre transformation interpolates between different conformal dimensions, as expected. In other words, the divergent local terms contain information about the transformed generating functional, and then they have to be taken into account when performing the Legendre transformation. In the particular situation in which the divergent local terms cancel out and the conformal dimension $\Delta_{-}(\varrho)$ arises, the addition of counterterms to the on-shell action is not required.

Finally, we have also shown that there exists one particular case which has no bulk counterpart, namely the one in which the conformal dimension reaches the unitarity bound and becomes independent of the effective mass. This puzzling phenomenon deserves further studies.

\section{Acknowledgements}

P.M. acknowledges financial support by FAPESP grant 01/05770-1. V.O.R. is partially supported by CNPq and PRONEX under contract CNPq 66.2002/1998-99.

\section{A Hypergeometric Functions ${ }_{2} \mathrm{~F}_{1}$}

$$
{ }_{2} F_{1}(a, b ; c ; x)=\frac{\Gamma(c)}{\Gamma(a) \Gamma(b)} \sum_{n \geq 0} \frac{\Gamma(a+n) \Gamma(b+n)}{\Gamma(c+n)} \frac{x^{n}}{n !} .
$$

i) For $c-a-b \notin \mathbf{Z}$

$$
\begin{aligned}
{ }_{2} F_{1}(a, b ; c ; x) & =\frac{\Gamma(c) \Gamma(c-a-b)}{\Gamma(c-a) \Gamma(c-b)}{ }_{2} F_{1}(a, b ; a+b-c+1 ; 1-x) \\
& +(1-x)^{c-a-b} \frac{\Gamma(c) \Gamma(a+b-c)}{\Gamma(a) \Gamma(b)} \\
& \times{ }_{2} F_{1}(c-a, c-b ; c-a-b+1 ; 1-x) .
\end{aligned}
$$

ii) For $c-a-b=0$

$$
{ }_{2} F_{1}(a, b ; c ; x)=-\frac{\Gamma(a+b)}{[\Gamma(a)]^{2}[\Gamma(b)]^{2}} \sum_{n \geq 0} \frac{\Gamma(a+n) \Gamma(b+n)}{[\Gamma(n+1)]^{2}}
$$




$$
\times \quad\left[\ln (1-x)+u_{n}(a, b, 0)\right](1-x)^{n},
$$

where

$$
\begin{gathered}
u_{n}(a, b, m)=\beta(n+a+m)+\beta(n+b+m)-\beta(n+1)-\beta(n+1+m), \\
\beta(z)=\frac{d}{d z} \ln \Gamma(z), \\
\beta(1)=-\gamma \quad \beta(n)=-\gamma+\sum_{p=1}^{n-1} \frac{1}{p} \quad(n=2,3, \cdots),
\end{gathered}
$$

and $\gamma$ is the Euler constant.

iii) For $c-a-b=m \quad(m=1,2, \cdots)$

$$
\begin{aligned}
{ }_{2} F_{1}(a, b ; c ; x) & =\frac{\Gamma(m) \Gamma(a+b+m) \Gamma(1-m)}{\Gamma(a) \Gamma(b) \Gamma(a+m) \Gamma(b+m)} \sum_{n=0}^{m-1} \frac{\Gamma(n+a) \Gamma(n+b)}{\Gamma(n+1) \Gamma(n+1-m)}(1-x)^{n} \\
& -(-1)^{m} \frac{\Gamma(a+b+m)}{\Gamma(a) \Gamma(b) \Gamma(a+m) \Gamma(b+m)} \sum_{n \geq 0} \frac{\Gamma(n+a+m) \Gamma(n+b+m)}{\Gamma(n+1) \Gamma(n+1+m)} \\
& \times\left[\ln (1-x)+u_{n}(a, b, m)\right](1-x)^{n+m} .
\end{aligned}
$$

iv) For $c-a-b=-m \quad(m=1,2, \cdots)$

$$
\begin{aligned}
{ }_{2} F_{1}(a, b ; c ; x) & =\frac{\Gamma(m) \Gamma(a+b-m) \Gamma(1-m)}{\Gamma(a) \Gamma(b) \Gamma(a-m) \Gamma(b-m)} \\
& \times \sum_{n=0}^{m-1} \frac{\Gamma(n+a-m) \Gamma(n+b-m)}{\Gamma(n+1) \Gamma(n+1-m)}(1-x)^{n-m} \\
& -(-1)^{m} \frac{\Gamma(a+b-m)}{\Gamma(a) \Gamma(b) \Gamma(a-m) \Gamma(b-m)} \sum_{n \geq 0} \frac{\Gamma(n+a) \Gamma(n+b)}{\Gamma(n+1) \Gamma(n+1+m)} \\
& \times\left[\ln (1-x)+v_{n}(a, b, m)\right](1-x)^{n}
\end{aligned}
$$


where

$$
v_{n}(a, b, m)=\beta(n+a)+\beta(n+b)-\beta(n+1)-\beta(n+1+m) .
$$

\section{B Results Obtained from the Generalized AdS/CFT Prescription}

In this appendix, we summarize the main results which are obtained through the generalized AdS/CFT prescription Eqs.(29-31), for Dirichlet and Types I and II mixed boundary conditions. Let us first consider the case when $\nu$ is not an integer. We begin by analyzing a generic result, and then we will specialize to each specific situation. In general, we find generating functionals for the boundary CFT's which are of the generic form

$$
\mathcal{F}\left[f_{\epsilon}\right]=-\mathcal{C} \int d^{d} x d^{d} y f_{\epsilon}(\vec{x}) f_{\epsilon}(\vec{y}) \frac{\epsilon^{-2 \Delta_{1}}}{|\vec{x}-\vec{y}|^{2 \Delta_{2}}}
$$

where $\mathcal{C}$ is a coefficient, $f_{\epsilon}$ is the classical background which encodes the information about the boundary CFT, $\Delta_{1}$ is to be related to the asymptotic behavior of $f_{\epsilon}$ and $\Delta_{2}$ corresponds to the conformal dimension of the boundary operator. Taking the limit

$$
\lim _{\epsilon \rightarrow 0} \epsilon^{-\Delta_{1}} f_{\epsilon}(\vec{x})=f_{0}(\vec{x})
$$

and using the AdS/CFT prescription

$$
\exp (-\mathcal{F}) \equiv\left\langle\exp \left(\int d^{d} x \mathcal{O}_{f}(\vec{x}) f_{0}(\vec{x})\right)\right\rangle
$$

we find the boundary two-point function

$$
\left\langle\mathcal{O}_{f}(\vec{x}) \mathcal{O}_{f}(\vec{y})\right\rangle=2 \mathcal{C} \frac{1}{|\vec{x}-\vec{y}|^{2 \Delta_{2}}}
$$

Then, the field $f_{\epsilon}$ has the asymptotic behavior $\epsilon^{\Delta_{1}}$, and the operator $\mathcal{O}_{f}$ has conformal dimension $\Delta_{2}$.

Now we are ready to present the main results obtained for the specific cases of Dirichlet and Types I and II mixed boundary conditions. We consider each case separately. Recall that, in particular, the unitarity bound $(d-2) / 2$ arises only for $d \geq 3$. 


\section{B.1 Dirichlet}

\begin{tabular}{c|c|c|c|c} 
& $\Delta_{1}$ & $\Delta_{2}$ & $\mathcal{C}$ & Divergences \\
\hline$f_{\epsilon}=\phi_{\epsilon}$ & $\Delta_{-}(\varrho)$ & $\Delta_{+}(\varrho)$ & $\frac{\nu(\varrho)}{\pi^{\frac{d}{2}}} \frac{\Gamma\left(\Delta_{+}(\varrho)\right)}{\Gamma(\nu(\varrho))}$ & YES \\
$f_{\epsilon}=\tilde{\phi}_{\epsilon}$ & $\Delta_{-}(\varrho)$ & $\Delta_{+}(\varrho)$ & $\frac{\nu(\varrho)}{\pi^{\frac{d}{2}}} \frac{1}{\left[\Delta_{-}(\varrho)-2 \varrho d\right]^{2}} \frac{\Gamma\left(\Delta_{+}(\varrho)\right)}{\Gamma(\nu(\varrho))}$ & YES
\end{tabular}

Table 1: Values of $\Delta_{1}, \Delta_{2}$ and $\mathcal{C}$ and presence of divergences when Eq. (84) is not satisfied.

\begin{tabular}{c|c|c|c|c} 
& $\Delta_{1}$ & $\Delta_{2}$ & $\mathcal{C}$ & Divergences \\
\hline$f_{\epsilon}=\phi_{\epsilon}$ & $\Delta_{-}(\varrho)$ & $\Delta_{+}(\varrho)$ & $\frac{\nu(\varrho)}{\pi^{\frac{d}{2}}} \frac{\Gamma\left(\Delta_{+}(\varrho)\right)}{\Gamma(\nu(\varrho))}$ & NO \\
$f_{\epsilon}=\tilde{\phi}_{\epsilon}$ & $\Delta_{+}(\varrho)$ & $\Delta_{-}(\varrho)$ & $\frac{1}{4 \pi^{\frac{d}{2}}} \frac{\Gamma\left(\Delta_{-}(\varrho)\right)}{\Gamma(1-\nu(\varrho))}$ & NO
\end{tabular}

Table 2: Values of $\Delta_{1}, \Delta_{2}$ and $\mathcal{C}$ and presence of divergences when Eqs.(20, 84) are satisfied.

\begin{tabular}{c|c|c|c|c} 
& $\Delta_{1}$ & $\Delta_{2}$ & $\mathcal{C}$ & Divergences \\
\hline$f_{\epsilon}=\phi_{\epsilon}$ & $\Delta_{-}(\varrho)$ & $\Delta_{+}(\varrho)$ & $\frac{\nu(\varrho)}{\pi^{\frac{d}{2}}} \frac{\Gamma\left(\Delta_{+}(\varrho)\right)}{\Gamma(\nu(\varrho))}$ & YES \\
$f_{\epsilon}=\tilde{\phi}_{\epsilon}$ & $\frac{d+2}{2}$ & $\frac{d-2}{2}$ & $(\nu(\varrho)-1) \frac{\Gamma\left(\frac{d-2}{2}\right)}{4 \pi^{\frac{d}{2}}}$ & NO
\end{tabular}

Table 3: Values of $\Delta_{1}, \Delta_{2}$ and $\mathcal{C}$ and presence of divergences when Eq. (84) is satisfied, but Eq.(20) is not. 


\section{B.2 Type I Mixed}

\begin{tabular}{c|c|c|c|c} 
& $\Delta_{1}$ & $\Delta_{2}$ & $\mathcal{C}$ & Divergences \\
\hline$f_{\epsilon}=\psi_{\epsilon}^{I}$ & $\Delta_{-}(\varrho)$ & $\Delta_{+}(\varrho)$ & $\frac{\nu(\varrho)}{\pi^{\frac{d}{2}}} \frac{1}{\left[\Delta_{-}(\varrho)+2 \varrho d\right]^{2}} \frac{\Gamma\left(\Delta_{+}(\varrho)\right)}{\Gamma(\nu(\varrho))}$ & YES \\
$f_{\epsilon}=\tilde{\psi}_{\epsilon}^{I}$ & $\Delta_{-}(\varrho)$ & $\Delta_{+}(\varrho)$ & $\frac{\nu(\varrho)}{\pi^{\frac{d}{2}}} \frac{\Gamma\left(\Delta_{+}(\varrho)\right)}{\Gamma(\nu(\varrho))}$ & YES
\end{tabular}

Table 4: Values of $\Delta_{1}, \Delta_{2}$ and $\mathcal{C}$ and presence of divergences when Eq. (86) is not satisfied.

\begin{tabular}{c|c|c|c|c} 
& $\Delta_{1}$ & $\Delta_{2}$ & $\mathcal{C}$ & Divergences \\
\hline$f_{\epsilon}=\psi_{\epsilon}^{I}$ & $\Delta_{+}(\varrho)$ & $\Delta_{-}(\varrho)$ & $\frac{1}{4 \pi^{\frac{d}{2}}} \frac{\Gamma\left(\Delta_{-}(\varrho)\right)}{\Gamma(1-\nu(\varrho))}$ & NO \\
$f_{\epsilon}=\tilde{\psi}_{\epsilon}^{I}$ & $\Delta_{-}(\varrho)$ & $\Delta_{+}(\varrho)$ & $\frac{\nu(\varrho)}{\pi^{\frac{d}{2}}} \frac{\Gamma\left(\Delta_{+}(\varrho)\right)}{\Gamma(\nu(\varrho))}$ & NO
\end{tabular}

Table 5: Values of $\Delta_{1}, \Delta_{2}$ and $\mathcal{C}$ and presence of divergences when Eqs.(20, 86) are satisfied.

\begin{tabular}{c|c|c|c|c} 
& $\Delta_{1}$ & $\Delta_{2}$ & $\mathcal{C}$ & Divergences \\
\hline$f_{\epsilon}=\psi_{\epsilon}^{I}$ & $\frac{d+2}{2}$ & $\frac{d-2}{2}$ & $(\nu(\varrho)-1) \frac{\Gamma\left(\frac{d-2}{2}\right)}{4 \pi^{\frac{d}{2}}}$ & NO \\
$f_{\epsilon}=\tilde{\psi}_{\epsilon}^{I}$ & $\Delta_{-}(\varrho)$ & $\Delta_{+}(\varrho)$ & $\frac{\nu(\varrho)}{\pi^{\frac{d}{2}}} \frac{\Gamma\left(\Delta_{+}(\varrho)\right)}{\Gamma(\nu(\varrho))}$ & YES
\end{tabular}

Table 6: Values of $\Delta_{1}, \Delta_{2}$ and $\mathcal{C}$ and presence of divergences when Eq.(86) is satisfied, but Eq.(20) is not. 


\section{B.3 Type II Mixed}

In this case, we also need to consider the additional constraint

$$
m=0
$$

\begin{tabular}{c|c|c|c|c} 
& $\Delta_{1}$ & $\Delta_{2}$ & $\mathcal{C}$ & Divergences \\
\hline$f_{\epsilon}=\psi_{\epsilon}^{I I}$ & $\Delta_{-}(0)$ & $\Delta_{+}(0)$ & $\frac{\nu(0)}{\pi^{\frac{d}{2}}} \frac{1}{\left[1-2 \lambda \Delta_{-}(0)\right]^{2}} \frac{\Gamma\left(\Delta_{+}(0)\right)}{\Gamma(\nu(0))}$ & YES \\
$f_{\epsilon}=\tilde{\psi}_{\epsilon}^{I I}$ & $\Delta_{-}(0)$ & $\Delta_{+}(0)$ & $\frac{\nu(0)}{\pi^{\frac{d}{2}}} \frac{1}{\left[\Delta_{-}(0)\right]^{2}} \frac{\Gamma\left(\Delta_{+}(0)\right)}{\Gamma(\nu(0))}$ & YES
\end{tabular}

Table 7: Values of $\Delta_{1}, \Delta_{2}$ and $\mathcal{C}$ and presence of divergences when Eqs. (88, 185) are not satisfied.

\begin{tabular}{c|c|c|c|c} 
& $\Delta_{1}$ & $\Delta_{2}$ & $\mathcal{C}$ & Divergences \\
\hline$f_{\epsilon}=\psi_{\epsilon}^{I I}$ & $\Delta_{+}(0)$ & $\Delta_{-}(0)$ & $\frac{1}{4 \pi^{\frac{d}{2}}}\left[\Delta_{-}(0)\right]^{2} \frac{\Gamma\left(\Delta_{-}(0)\right)}{\Gamma(1-\nu(0))}$ & NO \\
$f_{\epsilon}=\tilde{\psi}_{\epsilon}^{I I}$ & $\Delta_{-}(0)$ & $\Delta_{+}(0)$ & $\frac{\nu(0)}{\pi^{\frac{d}{2}}} \frac{1}{\left[\Delta_{-}(0)\right]^{2}} \frac{\Gamma\left(\Delta_{+}(0)\right)}{\Gamma(\nu(0))}$ & NO
\end{tabular}

Table 8: Values of $\Delta_{1}, \Delta_{2}$ and $\mathcal{C}$ and presence of divergences when Eqs.(20, 88) are satisfied.

\begin{tabular}{c|c|c|c|c} 
& $\Delta_{1}$ & $\Delta_{2}$ & $\mathcal{C}$ & Divergences \\
\hline$f_{\epsilon}=\psi_{\epsilon}^{I I}$ & $\frac{d+2}{2}$ & $\frac{d-2}{2}$ & $(\nu(0)-1)\left[\Delta_{-}(0)\right]^{2} \frac{\Gamma\left(\frac{d-2}{2}\right)}{4 \pi^{\frac{d}{2}}}$ & NO \\
$f_{\epsilon}=\tilde{\psi}_{\epsilon}^{I I}$ & $\Delta_{-}(0)$ & $\Delta_{+}(0)$ & $\frac{\nu(0)}{\pi^{\frac{d}{2}}} \frac{1}{\left[\Delta_{-}(0)\right]^{2}} \frac{\Gamma\left(\Delta_{+}(0)\right)}{\Gamma(\nu(0))}$ & YES
\end{tabular}

Table 9: Values of $\Delta_{1}, \Delta_{2}$ and $\mathcal{C}$ and presence of divergences when Eq.(88) is satisfied, but Eq.(20) is not. 


\begin{tabular}{c|c|c|c|c} 
& $\Delta_{1}$ & $\Delta_{2}$ & $\mathcal{C}$ & Divergences \\
\hline$f_{\epsilon}=\psi_{\epsilon}^{I I}$ & $\Delta_{-}(0)(=0)$ & $\Delta_{+}(0)(=d)$ & $\frac{d}{2 \pi^{\frac{d}{2}}} \frac{\Gamma(d)}{\Gamma\left(\frac{d}{2}\right)}$ & YES \\
$f_{\epsilon}=\tilde{\psi}_{\epsilon}^{I I}$ & $\frac{d+2}{2}$ & $\frac{d-2}{2}$ & $\frac{\Gamma\left(\frac{d}{2}\right)}{4 \pi^{\frac{d}{2}}}$ & NO
\end{tabular}

Table 10: Values of $\Delta_{1}, \Delta_{2}$ and $\mathcal{C}$ and presence of divergences when Eq.(185) is satisfied.

Now we consider the case of $\nu$ integer. For $\nu$ integer but non-zero, it can be shown, following a procedure analogous to the one in [7], that, after integrating over the momentum, the leading non-local terms are equal to the ones that we have found in the case of $\nu$ not integer, thus giving rise to the same boundary two-point functions. Besides, in addition to the usual divergent local terms, there arise logarithmic divergences, which correspond to conformal anomalies.

To finish, we present the results corresponding to the case $\nu=0$. As in the case of $\nu$ not integer, we begin by analyzing a generic result, and then we will specialize to each specific situation. For $\nu=0$, in general we find two different kinds of leading non-local terms. They have the generic form

$$
\mathcal{F}_{1}\left[f_{\epsilon}\right]=-\mathcal{C} \int d^{d} x d^{d} y f_{\epsilon}(\vec{x}) f_{\epsilon}(\vec{y}) \frac{\epsilon^{-d}}{l n^{2} \epsilon} \frac{1}{|\vec{x}-\vec{y}|^{d}}
$$

and

$$
\mathcal{F}_{2}\left[f_{\epsilon}\right]=-\mathcal{C} \int d^{d} x d^{d} y f_{\epsilon}(\vec{x}) f_{\epsilon}(\vec{y}) \frac{\epsilon^{-d}}{|\vec{x}-\vec{y}|^{d}}
$$

In the first one, we take the limit

$$
\lim _{\epsilon \rightarrow 0}\left(\epsilon^{\frac{d}{2}} \ln \epsilon\right)^{-1} f_{\epsilon}(\vec{x})=f_{0}(\vec{x}),
$$

which corresponds to the asymptotic behavior $x_{0}^{\frac{d}{2}} \ln x_{0}$. In the second one, the limit to be taken is 


$$
\lim _{\epsilon \rightarrow 0} \epsilon^{-\frac{d}{2}} f_{\epsilon}(\vec{x})=f_{0}(\vec{x})
$$

and this corresponds to the asymptotic behavior $x_{0}^{\frac{d}{2}}$. In both cases, we find the following boundary two-point function

$$
\left\langle\mathcal{O}_{f}(\vec{x}) \mathcal{O}_{f}(\vec{y})\right\rangle=2 \mathcal{C} \frac{1}{|\vec{x}-\vec{y}|^{d}},
$$

which corresponds to the conformal dimension $\frac{d}{2}$.

Now we are ready to present the main results obtained for the specific cases of Dirichlet and Types I and II mixed boundary conditions. We consider each case separately.

\section{B.4 Dirichlet}

\begin{tabular}{c|c|c} 
& $\mathcal{F}$ & $\mathcal{C}$ \\
\hline$f_{\epsilon}=\phi_{\epsilon}$ & $\mathcal{F}_{1}$ & $\frac{\Gamma\left(\frac{d}{2}\right)}{4 \pi^{\frac{d}{2}}}$ \\
$f_{\epsilon}=\tilde{\phi}_{\epsilon}$ & $\mathcal{F}_{1}$ & $\frac{1}{\left(\frac{d}{2}-2 \varrho d\right)^{2}} \frac{\Gamma\left(\frac{d}{2}\right)}{4 \pi^{\frac{d}{2}}}$
\end{tabular}

Table 11: Leading non-local terms and values of $\mathcal{C}$ when Eq.(84) is not satisfied.

\begin{tabular}{c|c|c} 
& $\mathcal{F}$ & $\mathcal{C}$ \\
\hline$f_{\epsilon}=\phi_{\epsilon}$ & $\mathcal{F}_{1}$ & $\frac{\Gamma\left(\frac{d}{2}\right)}{4 \pi^{\frac{d}{2}}}$ \\
$f_{\epsilon}=\tilde{\phi}_{\epsilon}$ & $\mathcal{F}_{2}$ & $\frac{\Gamma\left(\frac{d}{2}\right)}{4 \pi^{\frac{d}{2}}}$
\end{tabular}

Table 12: Leading non-local terms and values of $\mathcal{C}$ when Eq.(84) is satisfied. 


\section{B.5 Type I Mixed}

\begin{tabular}{c|c|c} 
& $\mathcal{F}$ & $\mathcal{C}$ \\
\hline$f_{\epsilon}=\psi_{\epsilon}^{I}$ & $\mathcal{F}_{1}$ & $\frac{1}{\left(\frac{d}{2}+2 \varrho d\right)^{2}} \frac{\Gamma\left(\frac{d}{2}\right)}{4 \pi^{\frac{d}{2}}}$ \\
$f_{\epsilon}=\tilde{\psi}_{\epsilon}^{I}$ & $\mathcal{F}_{1}$ & $\frac{\Gamma\left(\frac{d}{2}\right)}{4 \pi^{\frac{d}{2}}}$
\end{tabular}

Table 13: Leading non-local terms and values of $\mathcal{C}$ when Eq.(86) is not satisfied.

\begin{tabular}{c|c|c} 
& $\mathcal{F}$ & $\mathcal{C}$ \\
\hline$f_{\epsilon}=\psi_{\epsilon}^{I}$ & $\mathcal{F}_{2}$ & $\frac{\Gamma\left(\frac{d}{2}\right)}{4 \pi^{\frac{d}{2}}}$ \\
$f_{\epsilon}=\tilde{\psi}_{\epsilon}^{I}$ & $\mathcal{F}_{1}$ & $\frac{\Gamma\left(\frac{d}{2}\right)}{4 \pi^{\frac{d}{2}}}$
\end{tabular}

Table 14: Leading non-local terms and values of $\mathcal{C}$ when Eq.(86) is satisfied.

\section{B.6 Type II Mixed}

\begin{tabular}{|c|c|c|}
\hline & $\mathcal{F}$ & $\mathcal{C}$ \\
\hline$f_{\epsilon}=\psi_{\epsilon}^{I I}$ & $\mathcal{F}_{1}$ & $\frac{1}{(1-\lambda d)^{2}} \frac{\Gamma\left(\frac{d}{2}\right)}{4 \pi^{\frac{d}{2}}}$ \\
\hline$f_{\epsilon}=\tilde{\psi}_{\epsilon}^{I I}$ & $\mathcal{F}_{1}$ & $\left(\frac{2}{d}\right)^{2} \frac{\Gamma\left(\frac{d}{2}\right)}{d-\frac{d}{d}}$ \\
\hline
\end{tabular}

Table 15: Leading non-local terms and values of $\mathcal{C}$ when Eq.(88) is not satisfied. 


\begin{tabular}{|c|c|c|}
\hline & $\mathcal{F}$ & $\mathcal{C}$ \\
\hline$f_{\epsilon}=\psi_{\epsilon}^{I I}$ & $\mathcal{F}_{2}$ & $\left(\frac{d}{2}\right)^{2} \frac{\Gamma\left(\frac{d}{2}\right)}{4 \pi^{\frac{d}{2}}}$ \\
\hline$f_{\epsilon}=\tilde{\psi}_{\epsilon}^{I I}$ & $\mathcal{F}_{1}$ & $\left(\frac{2}{d}\right)^{2} \frac{\Gamma\left(\frac{d}{2}\right)}{4 \pi^{\frac{d}{2}}}$ \\
\hline
\end{tabular}

Table 16: Leading non-local terms and values of $\mathcal{C}$ when Eq.(88) is satisfied.

\section{References}

[1] J. M. Maldacena, "The Large N Limit of Superconformal Field Theories and Supergravity", Adv. Theor. Math. Phys. 2 (1998) 231; Int. J. Theor. Phys. 38 (1999) 1113, hep-th/9711200.

[2] E. Witten, "Anti De Sitter Space and Holography", Adv. Theor. Math. Phys. 2 (1998) 253, hep-th/9802150.

[3] S. S. Gubser, I. R. Klebanov and A. M. Polyakov, "Gauge Theory Correlators from Non-Critical String Theory", Phys. Lett. B428 (1998) 105, hep-th/9802109.

[4] D. Z. Freedman, S. D. Mathur, A. Matusis and L. Rastelli, "Correlation functions in the $C F T_{d} / A d S_{d+1}$ correspondence", Nucl. Phys. B546 (1999) 96, hepth/9804058.

[5] W. Mück and K. S. Viswanathan, "Conformal Field Theory Correlators from Classical Scalar Field Theory on $A d S_{d+1}$ ", Phys. Rev. D58 (1998) 041901, hepth/9804035.

[6] V. Balasubramanian, P. Kraus and A. Lawrence, "Bulk vs. Boundary Dynamics in Anti-de Sitter Spacetime", Phys. Rev. D59 (1999) 046003, hep-th/9805171.

[7] P. Minces and V. O. Rivelles, "Scalar Field Theory in the AdS/CFT Correspondence Revisited", Nucl. Phys. B572 (2000) 651, hep-th/9907079.

[8] M. Henningson and K. Sfetsos, "Spinors and the AdS/CFT correspondence", Phys. Lett. B431 (1998) 63, hep-th/9803251. 
[9] W. Mück and K. S. Viswanathan, "Conformal Field Theory Correlators from Classical Field Theory on Anti-de Sitter Space II. Vector and Spinor Fields", Phys. Rev. D58 (1998) 106006, hep-th/9805145.

[10] M. Henneaux, "Boundary terms in the AdS/CFT correspondence for spinor fields", Proceedings of the International Workshop ISMP (Tbilissi, September 1998), hep-th/9902137.

[11] G. E. Arutyunov and S. A. Frolov, "On the origin of supergravity boundary terms in the AdS/CFT correspondence", Nucl. Phys. B544 (1999) 576, hep-th/9806216.

[12] P. Minces and V. O. Rivelles, "Chern-Simons Theories in the AdS/CFT Correspondence", Phys. Lett. B455 (1999) 147, hep-th/9902123.

[13] H. O. Girotti and V. O. Rivelles, "Gauge Dependence in the AdS/CFT Correspondence", Int. Jour. Mod. Phys. A15 (2000) 4379, hep-th/9910017.

[14] A. Volovich, "Rarita-Schwinger Field in the AdS/CFT Correspondence", JHEP 9809 (1998) 022, hep-th/9809009.

[15] A. S. Koshelev and O. A. Rytchkov, "Note on the Massive Rarita-Schwinger Field in the AdS/CFT correspondence", Phys. Lett. B450 (1999) 368, hep-th/9812238.

[16] P. Matlock and K. S. Viswanathan, "The AdS/CFT Correspondence for the Massive Rarita-Schwinger Field", Phys. Rev. D61 (2000) 026002, hep-th/9906077.

[17] H. Liu and A. A. Tseytlin, " $\mathrm{D}=4$ Super Yang Mills, $\mathrm{D}=5$ gauged supergravity and $\mathrm{D}=4$ conformal supergravity", Nucl. Phys. B533 (1998) 88, hep-th/9804083.

[18] W. Mück and K. S. Viswanathan, "The Graviton in the AdS-CFT correspondence: Solution via the Dirichlet Boundary value problem", hep-th/9810151.

[19] A. Polishchuk, "Massive symmetric tensor field on AdS", JHEP 9907 (1999) 007, hep-th/9905048.

[20] G. E. Arutyunov and S. A. Frolov, "Antisymmetric tensor field on $A d S_{5}$ ", Phys. Lett. B441 (1998) 173, hep-th/9807046.

[21] W. S. l'Yi, "Correlators of currents corresponding to the massive $p$-form fields in AdS/CFT correspondence", Phys. Lett. B448 (1999) 218, hep-th/9811097. 
[22] M. Henningson and K. Skenderis, "The Holographic Weyl Anomaly", JHEP 9807 (1998) 023, hep-th/9806087.

[23] I. R. Klebanov and E. Witten, "AdS/CFT Correspondence and Symmetry Breaking", Nucl. Phys. B556 (1999) 89, hep-th/9905104.

[24] S. Nojiri and S. D. Odintsov, "Conformal Anomaly for Dilaton Coupled Theories from AdS/CFT Correspondence", Phys. Lett. B444 (1998) 92, hep-th/9810008.

[25] S. Nojiri, S. D. Odintsov and S. Ogushi, "Finite Action in d5 Gauged Supergravity and Dilatonic Conformal Anomaly for Dual Quantum Field Theory", Phys. Rev. D62 (2000) 124002, hep-th/0001122.

[26] V. Balasubramanian and P. Kraus, "A Stress Tensor for Anti-de Sitter Gravity", Comm. Math. Phys. 208 (1999) 413, hep-th/9902121.

[27] R. Emparan, C. V. Johnson and R. C. Myers, "Surface Terms as Counterterms in the AdS/CFT Correspondence", Phys. Rev. D60 (1999) 104001, hep-th/9903238.

[28] W. Mück and K.S. Viswanathan, "Counterterms for the Dirichlet Prescription of the AdS/CFT Correspondence", hep-th/9905046.

[29] A. Petkou and K. Skenderis, "A non-renormalization theorem for conformal anomalies", Nucl. Phys. B561 (1999) 100, hep-th/9906030.

[30] S. de Haro, K. Skenderis and S. Solodukhin, "Holographic Reconstruction of Spacetime and Renormalization in the AdS/CFT Correspondence", Comm. Math. Phys. 217 (2001) 595, hep-th/0002230.

[31] E. T. Akhmedov, "A remark on the AdS/CFT correspondence and the renormalization group flow", Phys. Lett B442 (1998) 152, hep-th/9806217.

[32] E. Alvarez and C. Gomez, "Geometric Holography, the Renormalization Group and the c-Theorem", Nucl. Phys. B541 (1999) 441, hep-th/9807226.

[33] D. Z. Freedman, S. S. Gubser, K. Pilch and N. P. Warner, "Renormalization Group Flows from Holography-Supersymmetry and a c-Theorem", Adv. Theor. Math. Phys. 3 (1999) 363, hep-th/9904017.

[34] J. de Boer, E. Verlinde and H. Verlinde, "On the Holographic Renormalization Group", JHEP 0008 (2000) 003, hep-th/9912012. 
[35] P. Breitenlohner and D. Z. Freedman, "Stability in Gauged Extended Supergravity", Ann. Phys. 144 (1982) 249.

[36] P. Breitenlohner and D. Z. Freedman, "Positive Energy in Anti-de Sitter Backgrounds and Gauged Extended Supergravity", Phys. Lett. B115 197.

[37] H. Boschi-Filho and N. Braga, "Quantum fields in anti de Sitter spacetime and degrees of freedom in the bulk/boundary correspondence", Phys. Lett. B505 (2001) 263, hep-th/0009039.

[38] H. Boschi-Filho and N. Braga, "Field spectrum and degrees of freedom in AdS/CFT correspondence and Randall Sundrum model", Nucl. Phys. B608 (2001) 319, hep-th/0012196.

[39] H. Boschi-Filho and N. Braga, "Bulk versus boundary quantum states", hepth/0106108.

[40] L. Mezincescu and P. K. Townsend, "Stability at a Local Maximum in Higher Dimensional Anti-de Sitter Space and Applications to Supergravity", Ann. Phys. 160 (1985) 406.

[41] S. J. Avis, C. J. Isham and D. Storey, "Quantum Field Theory in Anti-de Sitter Space-Time", Phys. Rev. D18 (1978) 3565.

[42] V. K. Dobrev, "Intertwining Operator Realization of the AdS/CFT Correspondence", Nucl. Phys. B553 (1999) 559, hep-th/9812194.

[43] L. F. Abbott and S. Deser, "Stability of Gravity with a Cosmological Constant", Nucl. Phys. B195 (1982) 76.

[44] D. V. Fursaev, "Energy, Hamiltonian, Noether Charge, and Black Holes", Phys. Rev. D59 (1999) 064020, hep-th/9809049.

[45] V. O. Soloviev, "Boundary values as Hamiltonian variables. I. New Poisson brackets", J. Math. Phys. 34 (1993) 5747, hep-th/9305133.

[46] K. Bering, "Putting an Edge to the Poisson Bracket", J. Math. Phys. 41 (2000) 7468 , hep-th/9806249.

[47] G. W. Gibbons and S. W. Hawking, "Action Integrals and Partition Functions in Quantum Gravity", Phys. Rev. D15 (1977) 2752. 
[48] A. O. Barvinsky and S. N. Solodukhin, "Non-minimal coupling, boundary terms and renormalization of the Einstein-Hilbert action and black hole entropy", Nucl. Phys. B479 (1996) 305, gr-qc/9512047. 